\title{
Tunable colloids: control of colloidal phase transitions with tunable interactions
}

\author{
Anand Yethiraj \\ Received 20th March 2007, Accepted 1st June 2007 \\ First published as an Advance Article on the web 5th July 2007 \\ DOI: $10.1039 / \mathrm{b} 704251 \mathrm{p}$
}

Systems of spherical colloidal particles mimic the thermodynamics of atomic crystals. Control of interparticle interactions in colloids, which has recently begun to be extensively exploited, gives rise to rich phase behaviours as well as crystal structures with nanoscale and micron-scale lattice spacings. This provides model systems in which to study fundamental problems in condensed matter physics, such as the dynamics of crystal nucleation and melting, and the nature of the glass transition, at experimentally accessible lengthscales and timescales. Tunable control of these interactions provides reversible control. This will enable quantitative studies of phase transition kinetics as well as the creation of advanced materials with switchability of function and properties.

\section{Introduction}

The self-assembly of spherical colloids mimics the thermodynamics of atomic crystals and has been studied for several decades. $^{1-3}$ Although self-assembly in colloids with shortrange and long-range interactions has been well-studied, the ability to control the colloidal interparticle interactions experimentally has recently begun to be extensively exploited. Phase transitions from an isotropic fluid phase to crystal and glass, ${ }^{3}$ as well as a two-dimensional hexatic phase, ${ }^{4}$ have been observed as a function of density. Fluid-fluid transitions, crystal-crystal martensitic transitions, ${ }^{5,6}$ a liquid-crystal-like phase, $^{7}$ as well as dynamics of crystallization ${ }^{8-12}$ and melting ${ }^{7}$ have been observed, with recent developments extending the analogy further to colloidal molecules. ${ }^{13-15}$ Reversible control of interparticle interactions, or tunability, lends itself to cycling through a phase transition several times, leading to better

Department of Physics and Physical Oceanography, Memorial University of Newfoundland, St. John's, NL, Canada.

E-mail: anand@physics.mun.ca

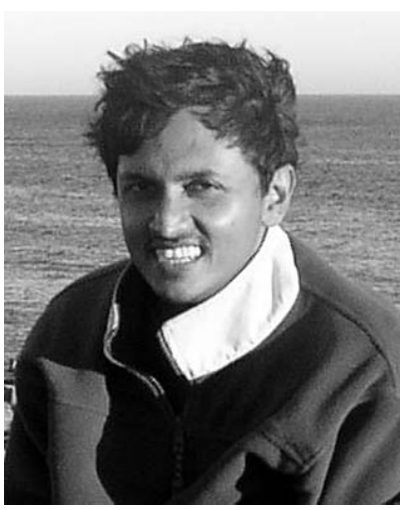

Anand Yethiraj
Anand Yethiraj's research interests focus on the study of all forms of soft matter via optical microscopies and NMR spectroscopy. He completed a PhD at Simon Fraser University, Canada. He has done post-doctoral research at the FOM Institute AMOLF and Utrecht University in the Netherlands, and the Chemistry Department at the University of British Columbia. He is currently an assistant professor at the Department of Physics and

Physical Oceanography at the Memorial University in St John's, NL, Canada. quantitative studies of phase-transition kinetics. Tunability also lends itself well to the possibility of creating advanced materials whose function and properties can be switched, i.e. controlled reversibly. This review focuses on passive and active (tunable) control of interactions in model colloidal suspensions consisting of spherical solid particles.

Colloidal particles are in constant Brownian motion caused by the molecular nature of the surrounding fluid medium $^{1,16}$ and the self-assembly that results from this access to configurational entropy has immense structural diversity. ${ }^{3,17}$ A variety of techniques such as optical microscopy, ${ }^{2}$ static and dynamic light scattering, ${ }^{3}$ laser scanning confocal microscopy, ${ }^{18}$ small angle X-ray scattering ${ }^{19}$ and small-angle neutron scattering ${ }^{20}$ have been used to study quiescent colloidal suspensions. In dense colloidal suspensions, multiple scattering is important, except when the particle and solvent refractive indices are carefully matched. Such refractive index matching is an imperative in confocal microscopy as well as conventional light scattering. Diffusing wave spectroscopy ${ }^{21}$ and two-colour dynamic light scattering ${ }^{22}$ are techniques designed specifically to address the issue of multiple scattering in colloids. In addition, bulk rheology (as well as microrheology ${ }^{23}$ ) has probed colloidal response to shear.

Colloidal particles interact with each other via the entropic excluded volume interaction as well as in several other ways: for example, long-range electrostatic interactions (controlled by charge on the spheres), short-ranged van der Waals interaction, and external electromagnetic and gravitational fields. In addition, one cannot neglect either hydrodynamic interactions or the presence of surfaces. In the absence of all interactions other than that of excluded volume, colloids behave like perfect "hard spheres".

Kirkwood, Alder, and coworkers ${ }^{24-26}$ first predicted that hard spheres would form an ordered phase well-below the absolute close-packing limit of $\phi=0.74$. Experiments agree with computer simulations that the phase behaviour of hard spheres $^{3,25-28}$ includes a fluid phase at low particle volume fractions $\phi$ and fluid-solid coexistence in the range $0.494<\phi$ 
$<0.545$. In addition an amorphous phase that is identified as the glass phase is observed above $\phi=0.58 .^{3}$

The presence of additional interactions makes the phase behaviour even richer. The study of colloids as model atoms and molecules allows one to probe mechanisms involved in complex phenomena such as crystallization, melting and the glass transition. The study of colloidal phase behaviour has benefited greatly from the synergism of experiments with molecular dynamics and Monte Carlo computer simulations. The simulation of systems exhibiting long-range interparticle interactions such as the electrostatic, dipolar and hydrodynamic interactions is inherently difficult. Experimentally, perhaps the most frustrating aspect has been the dearth of convenient tuning parameters to traverse these rich phase diagrams in a single sample. This review focuses on current progress on tunable colloids - the ability to cycle reversibly through a phase transition.

A control of interactions is also of interest if one wishes to control colloid microstructure with a view to material science applications; of which many are being explored: photonic band-gap materials, ${ }^{29}$ electro-rheological fluids ${ }^{30,31}$ and patterned magnetic materials. ${ }^{32}$ Tunable control of interactions will also enable the creation of advanced materials with switchable functionality.

\section{Control of interactions}

The control of material parameters is crucially important to the experimental study of colloids as model atoms and molecules. Controlling colloidal interactions alters the interplay between energetics and entropy in the colloidal free energy, and thus alters the equilibrium structures observed. Different physical effects give rise to interactions in colloids. Their relative importance may be expressed in the form of lengths and dimensionless numbers (Table 1).

First we consider gravity, hydrodynamic, depletion and electrostatic interactions. In current versions of experiments, these interactions are indeed controllable, but not reversibly so in one sample. Effective interactions, such as those induced by patterned and unpatterned surfaces, are extremely important in colloids, but are not discussed here. Surface-induced interactions afford the immensely exciting prospect of creating colloidal structures that are not structures preferred in the bulk ("template-induced crystallization",33), as well as controlling orientations of structures that are preferred in the bulk. Readers are referred to ref. 10, 33-36 and a review (ref. 37).

\subsection{Gravity}

The colloidal thermodynamic analogy is predicated on the importance of Brownian motion. Brownian motion forces the colloidal particles to sample configuration space efficiently and makes ensemble averages and effective free energies meaningful. Gravity is a long-ranged interaction that is always important except in Space (Fig. 1A). On Earth, it can be weak or strong depending on colloid size and density. Its importance in a colloidal system may be characterized by a "gravitational height" $h_{\text {grav }}$ (see Table 1). When the "scaled gravitational height" $\frac{h_{\text {grav }}}{2 a}$ (Fig. 1B) becomes comparable to or smaller than
Table 1 Lengths and dimensionless numbers that express the relative importance of some relevant (gravitational, electrostatic, electric and magnetic dipolar, hydrodynamic) physical interactions in colloids. Parameters used are the electron charge $e$, ion valency $z$, ion concentration in the bulk $c_{0}^{*}$, fluid viscosity $\eta_{\mathrm{f}}$, the shear rate $\dot{\gamma}$, electric field strength $E_{0}$, the particle and fluid dielectric constant $\varepsilon_{\mathrm{p}}$ and $\varepsilon_{\mathrm{f}}$ and dielectric mismatch parameter $\beta=\frac{-1+\varepsilon_{\mathrm{p}} / \varepsilon_{\mathrm{f}}}{2+\varepsilon_{\mathrm{p}} / \varepsilon_{\mathrm{f}}}$, the particle-fluid density mismatch $\Delta \rho=\left(\rho_{\mathrm{p}}-\rho_{\mathrm{f}}\right)$, the particle radius $a$, and the gravitational acceleration $g$. The Mason and Peclet numbers are relevant even in a quiescent suspension because colloids are constantly in motion: here one may replace $\dot{\gamma}$ with $\frac{v}{L}$ where $v$ is a characteristic particle velocity and $L$ a typical length (often the particle radius $a$ )

\begin{tabular}{lll}
\hline Lengths and Numbers & Formula & Physical Effects \\
\hline Gravitational height & $h_{\text {grav }}=\frac{k_{\mathrm{B}} T}{\frac{4}{3} \pi a^{3} \Delta \rho g}$ & $\begin{array}{c}\text { Thermal- } \\
\text { gravitational }\end{array}$ \\
Debye-Hückel length & $\kappa^{-1}=\sqrt{\frac{\varepsilon_{0} \varepsilon_{\mathrm{f}} k_{\mathrm{B}} T}{2 e^{2} z^{2} c_{0}^{*}}}$ & $\begin{array}{c}\text { Electrostatic } \\
\text { screening }\end{array}$ \\
Colloid capillary length & $\xi_{\mathrm{cap}}=\sqrt{\frac{k_{\mathrm{B}} T / a^{2}}{\Delta \rho g}}$ & $\begin{array}{c}\text { Interfacial tension- } \\
\text { gravitational }\end{array}$ \\
Lambda parameter & $\Lambda=\frac{\pi \varepsilon_{0} \varepsilon_{\mathrm{f}} a^{3} \beta^{2} E_{0}^{2}}{2 k_{\mathrm{B}} T}$ & $\begin{array}{c}\text { Electric dipolar- } \\
\text { thermal }\end{array}$ \\
Gamma parameter & $\Gamma=\frac{\mu_{0}}{4 \pi}(\pi n)^{3 / 2}\left(\chi_{\mathrm{eff}} B\right)^{2}$ & $\begin{array}{c}\text { Magnetic dipolar- } \\
\text { thermal }\end{array}$ \\
Mason number & $M n=\frac{k_{\mathrm{B}} T}{\varepsilon_{0} \varepsilon_{\mathrm{f}} \beta^{2} E_{0}^{2}}$ & $\begin{array}{c}\text { Hydrodynamic- } \\
\text { electric dipolar }\end{array}$ \\
Peclet number & $P e=\frac{3 \pi a^{3} \eta_{\mathrm{f}} \dot{\gamma}}{k_{\mathrm{B}} T}$ & $\begin{array}{c}\text { Hydrodynamic- } \\
\text { thermal }\end{array}$ \\
\hline
\end{tabular}

unity, non-Brownian effects become important. For typical colloidal systems studied via light scattering, ${ }^{38-40}$ $10^{1}<\frac{h_{\text {grav }}}{2 a}<10^{5}$, while via optical microscopy $10^{-2}<\frac{h_{\text {grav }}}{2 a}<10^{3}$. Only when $\frac{h_{\text {grav }}}{2 a} \gg 1$ can the gravitational interaction be explicitly ignored.

2.1.1 "Zeroing" gravity. In a particulate suspension, the relative effect of gravity increases with particle size. In order to be model atoms, colloids must be studied in situations where gravity does not play a role. Matching the density of the particles and solvent can give rise to effective "milligravity". The effect of gravity can be further reduced in a time-averaged sense simply by rotation of the sample (about an axis perpendicular to the direction of the gravitational force), if a timescale exists that is simultaneously slow enough not to introduce dynamical forces and fast enough that a particle in suspension is static on the timescale of one rotation. ${ }^{41}$ Colloids in Space experience microgravity conditions. Surprising differences have been observed between milligravity and microgravity experiments. Dendritic growth of colloidal crystals is inhibited on earth (in milligravity), but not in Space (in microgravity), presumably because the terrestrial weight of the wispy dendritic arms causes them to shear-melt ${ }^{42}$ (Fig. 1A). Colloidal suspensions at densities up to $\phi=0.62$ (well into the glassy region on Earth, which begins at $\phi=0.58$ ) 


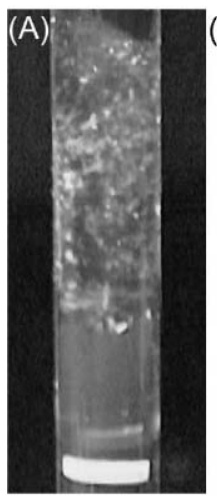

(B)

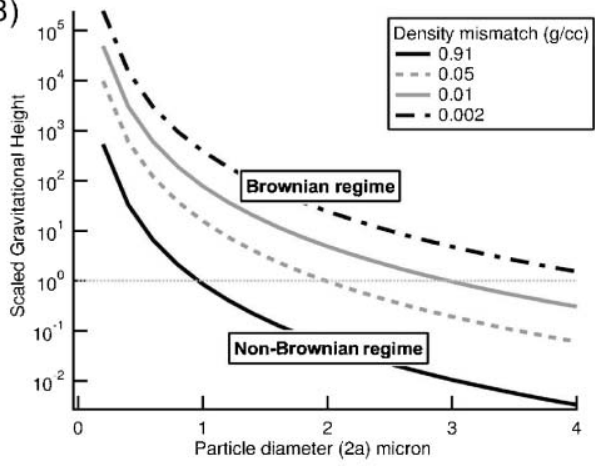

(C)

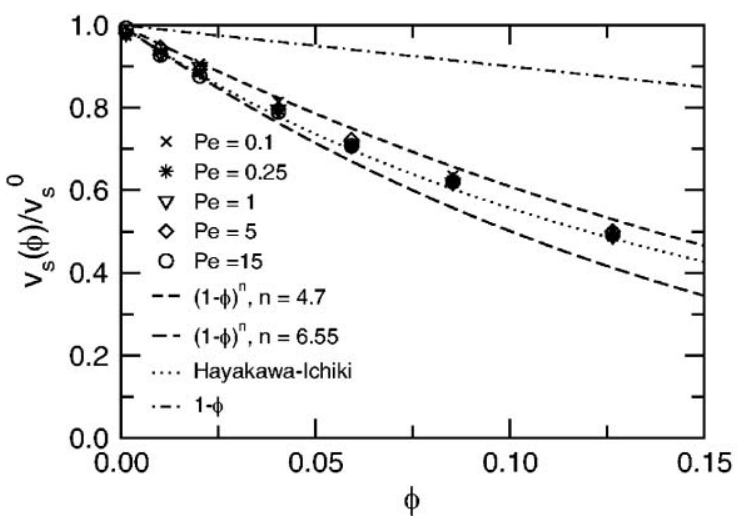

Fig. 1 Gravity. (A) With comparable samples and true microgravity conditions, colloids crystallize in Space (top half of image, sharp white patches are Bragg reflections) but are glassy on Earth (bottom half of image, diffuse grey above the white stir bar are the colloidal glass) ${ }^{12,42}$ (see text). (B) In many experimental systems, gravitational interactions are implicitly relevant. One measure of the advent of the nonBrownian regime in colloids is the scaled gravitational height $\left(\frac{h_{\text {grav }}}{2 a}\right)$ as it approaches unity from below. Shown are typical values for varying degrees of density matching, reflecting real systems such as silica and polystyrene in aqueous suspension $(0.91$ and $0.002-0.05$, respectively) and PMMA in non-aqueous suspensions (0.002-0.05) for different particle diameters. Optimal density matching gives effective "milligravity". (C) A crossover to non-Brownian behaviour is expected at large Peclet number. Computer simulations show that average colloidal sedimentation velocities exhibit strong hydrodynamic effects but are yet surprisingly insensitive to the Peclet number. ${ }^{48}$ Reprinted with permission from ref. 48. Copyright (2004) by the American Physical Society. http://link.aps.org/abstract/PRL/v93/e220601.

are observed to crystallize in Space. ${ }^{42}$ In fact, crystals formed in Space survive the space-shuttle's re-entry into earth, but can be easily shear melted on earth with a stir bar, top half of the sample shown) whereupon they remain glassy indefinitely! The sharp colours in the top half of the sample shown in Fig. 1A correspond to the Bragg reflections from a polycrystalline colloidal suspension, while the diffuse colours from the bottom half correspond to diffuse scattering from a colloidal glass. Moreover, in microgravity, the equilibrium crystal structure just above the melting volume fraction $(\phi=$ 0.545 ) is purely random hexagonal close packed (rhcp) (at terrestrial glassy densities) but face-centred cubic (fcc) when crystallized slowly in the coexistence region. ${ }^{12}$ This is consistent with computer simulations ${ }^{43,44}$ where the entropy difference between fcc and hcp crystal structures was found to be small $\left(\approx 10^{-3} k_{\mathrm{B}}\right)$.

2.1.2 Sedimentation: hydrodynamics. Colloid dynamics ${ }^{45}$ presents additional challenges, because of the range of timescales that are simultaneously important. The shortest timescale taken into account in simulations is that of diffusion of molecular velocity in an incompressible fluid medium from one colloidal macro-particle to another-this is typically on the order of $0.01-1 \mu \mathrm{s}$. The longest timescale is that of significant colloid motion - this is typically on the millisecond to second timescale.

Even when particles in fluid suspension interact as hard spheres, there always exists a fluid-mediated interaction. A moving sphere drags and displaces fluid. This resulting fluid flow affects other spheres. This is the hydrodynamic interaction in colloids, important in equilibrium conditions and dominant in non-equilibrium situations such as the sedimentation due to gravity.

As colloidal suspensions are made more concentrated the sedimentation velocity decreases ${ }^{46}$ as $v(\phi)=v^{0}(1-A \phi)$ where $v, v^{0}$, are sedimentation velocities at finite density and infinite dilution, and $\phi$ is the density (particle volume fraction). The prefactor $A$ is unity in the absence of hydrodynamic interactions and predicted to be 6.55 when hydrodynamics are taken into account at lowest order. ${ }^{46}$

A surprising computer simulation result ${ }^{48}$ finds (Fig. 1C) that the effect of hydrodynamic interactions on average sedimentation velocity appears not to change at all as the Peclet number is varied from $P e=0.1$ (hydrodynamic forces an order of magnitude smaller than thermal forces) to $P e=15$ (the reverse). Higher order corrections (as accounted for by ref. 47) give rise to a functional form (dotted line in Fig. 1C) that is quantitatively consistent with the simulation curve. An experimental test of the Peclet number insensitivity is awaited.

There exists a paradoxical theoretical result in colloidal hydrodynamics $^{49}$ (see ref. 50 for a review): velocity fluctuations in settling colloidal suspensions grow (and diverge) with the container size if the particles in the suspension are randomly distributed. Experimental work to measure velocity correlations have used a variety of imaging, spectroscopy and scattering techniques. ${ }^{51-54}$ Most studies found no evidence for the predicted divergence. An analog of the electrostatic Debye screening mechanism ${ }^{55,56}$ has been invoked theoretically (employing some form of non-random suspension structure) to suppress this divergence. It has been suggested that rearrangements in the particle structure, either due to "stratification" 57 or the formation of "blobs", rescue the system from this divergence by somehow suppressing the divergence. Also it has been shown that electrostatic interactions might screen hydrodynamic interactions in dense Brownian colloidal suspensions. ${ }^{58}$

However, it has been pointed out ${ }^{59}$ that common to all these experiments is the fact that while the system size perpendicular to the sedimentation direction was varied, the smallest dimension was along the sedimentation direction and typically no more than 50 particle diameters. Thus, no conclusive statement has yet been made about the effect of the vertical boundary on sedimentation. 


\subsection{Effective interactions arising from entropic effects}

Polymers can induce entropic effective attractive interactions among colloids due to excluded volume effects. When particle separation is small, the piece of any polymer chain in the gap is highly constrained in its possible configurations, resulting in a depletion of polymer segments from the gap. This depletion results in an effective colloid-colloid attraction. The range of this depletion interaction depends on the size of the polymer; the strength is a function of the polymer concentration. The interaction potential between a free colloidal sphere and a wall in the presence of non-charged polymer chains has been measured using total internal reflection, ${ }^{60}$ and this attractive potential was found to be strongly dependent on polymer concentration in accordance with an entropic mechanism.

The occurrence of polymer-induced colloidal fluid-solid and fluid-fluid phase separation was predicted by Gast et al. ${ }^{61}$ using the Asakura-Oosawa (AO) potential. In AO-like models ${ }^{62}$ the polymers interact with the colloids effectively as hard spheres with radius $R_{\mathrm{g}}$. Theory, ${ }^{61,63}$ and experiment ${ }^{64-66}$ are consistent with a phase diagram with fluid-solid phase boundaries at $R_{\mathrm{g}} / R<0.3$, with the solid phase being disrupted at larger size ratios, resulting in a phase diagram exhibiting a fluid-fluid phase transition as a function of colloid or polymer concentration.

Ramakrishnan et al. $^{67}$ mapped out the phase diagram of colloid-polymer mixtures as a function of polymer concentration $c_{\mathrm{p}}$ and colloid packing fraction $\phi$ for different size asymmetry ratios $R_{\mathrm{g}} / R$ (Fig. $2 \mathrm{~A}$ ). The qualitative nature of the phase diagram changed as $R_{\mathrm{g}} / R$ was varied over a wide range ( $\approx 0.03$ to 1.4 ). A fluid-gel transition was observed at $R_{\mathrm{g}} / R=$ 0.026 , and a fluid-solid phase boundary for $R_{\mathrm{g}} / R=0.115$, while for $R_{\mathrm{g}} / R>0.377$, a fluid-fluid phase boundary was observed. In general, suspension miscibility was found to improve with increasing $R_{\mathrm{g}} / R$. It is to be noted that the position of the gel boundary has a dependence on the influence of gravity (with gelation being suppressed in time-averaged zero gravity ${ }^{68,69}$ ).

A direct microscopic visualization of the physics is always instructive. Aarts et al. $^{70}$ explore the consequences of a colloidal gas-liquid transition: capillary waves are observed (Fig. 2B) due to the existence of gas-liquid surface tension: the interface gets rougher on approaching the critical point.

Pham et al. ${ }^{71}$ uncovered experimentally a remarkable phase diagram (predicted earlier by mode coupling theory ${ }^{72}$ and computer simulation ${ }^{73}$ ) consisting of two distinct glass phases, termed the "attractive" and the "repulsive" glass.

The normal repulsive glass is the same as the hard-sphere glass. Here the system becomes non-ergodic because the caging of particles by the neighbours prevents long-range particle motion. ${ }^{74}$ Turning on the attractive interaction reduces the average inter-particle spacing and induces a fluid phase. Reentrant glassy behaviour is expected ${ }^{72}$ when the width of the attractive potential is much shorter than the hard-core diameter.

Experimentally, control of short-range attractions is achieved by varying the polymer concentration. Fig. $2 \mathrm{C}$ shows dynamic structure factors as a function of time for five samples A-E with increasing polymer concentration (corresponding to increasing attractions). The inflection at $t=0.1 \mathrm{~s}$ in sample A corresponds to the (normal) glassy plateau in the presence of hard-sphere repulsions. Samples B and C show no glassy behaviour (no inflection point), characteristic of a fluid with short range attractions. Samples D and E once again show an inflection point corresponding to glassy behaviour, this time due to attractions. Since the total volume fraction is unchanged, the attractions create space for long-range motion and the glass melts. However, at high enough strengths, structural arrest reoccurs, this time due to bonding introduced by the strong inter-particle attractions. Direct imaging of this system via coherent anti-Stokes Raman (CARS) microscopy shows $^{75}$ that particles in the repulsive glasses exhibit cage rattling and escape, in contrast with the attractive glasses where cage escape is rarer, although more dramatic. Gel formation in colloid-polymer mixtures, which can occur by spinodal decomposition into colloid-rich and colloid-poor regions, also exhibits a local glass transition in the colloid-rich region. ${ }^{76}$ A unified understanding of gels and glasses will hopefully emerge from the colloid-polymer studies.

Two-component systems consisting of small and large spheres can also lead to entropic effects that can be interpreted as the small spheres modifying effective interactions of the large spheres. The effective pair potential between the large spheres has an attractive minimum at short distances, and an oscillatory part that is due to the liquid-like structure of the small spheres. ${ }^{77,78}$ Phase behaviour has been studied via computer simulation for a wide range of size ratios. ${ }^{79}$ Stable, isostructural solid-solid as well as fluid-fluid transitions were seen (the latter were metastable with respect to a fluid-solid transition). Phase separation has indeed been observed experimentally. ${ }^{80-82}$ The phase diagrams of binary hard spheres with size ratios between 2 and 12 were experimentally determined. ${ }^{80,81}$ Fluid-solid coexistence was observed, but no two-fluid coexistence was observed, contrary to expectations. ${ }^{83}$ Imhof et al. $^{84}$ have studied a binary colloidal mixture of size ratio $\approx 1: 9$ where fluid-solid phase coexistence, as well as a glassy phase with mobile small spheres, was also seen. For well-chosen sizes and packing fractions, phase separation is replaced by commensurate packing into a single-phase superlattice crystal. Superlattice $A B_{2}$ and $A B_{13}$ (also referred to as $L S_{2}$ and $L S_{13}$ with $L$ and $S$ standing for large and small) structures were observed at radius ratios close to $0.6 .^{85}$ While these superlattice structures comprise only a very small portion of the binary sphere phase diagram, they have immense application possibilities such as the creation of large-area binary photonic crystals. ${ }^{86}$

Finally, the depletion potential of sphere mixtures - where the "small" component is a mixture of spheres that are between 0.1 and 0.5 the radius of the large component ${ }^{87}$ show less-pronounced oscillatory structure as compared to simple binary mixtures, suggesting that this more complicated mixture might, perhaps, be a simpler way of inducing shortrange attractive interactions.

\subsection{Electrostatic interactions}

The electrostatic interaction is long-ranged (colloidal dust particles in air can be attracted at millimeter distances) and its strength and sign can be controlled from weak to very strong (several hundreds of $k_{\mathrm{B}} T$ ). 

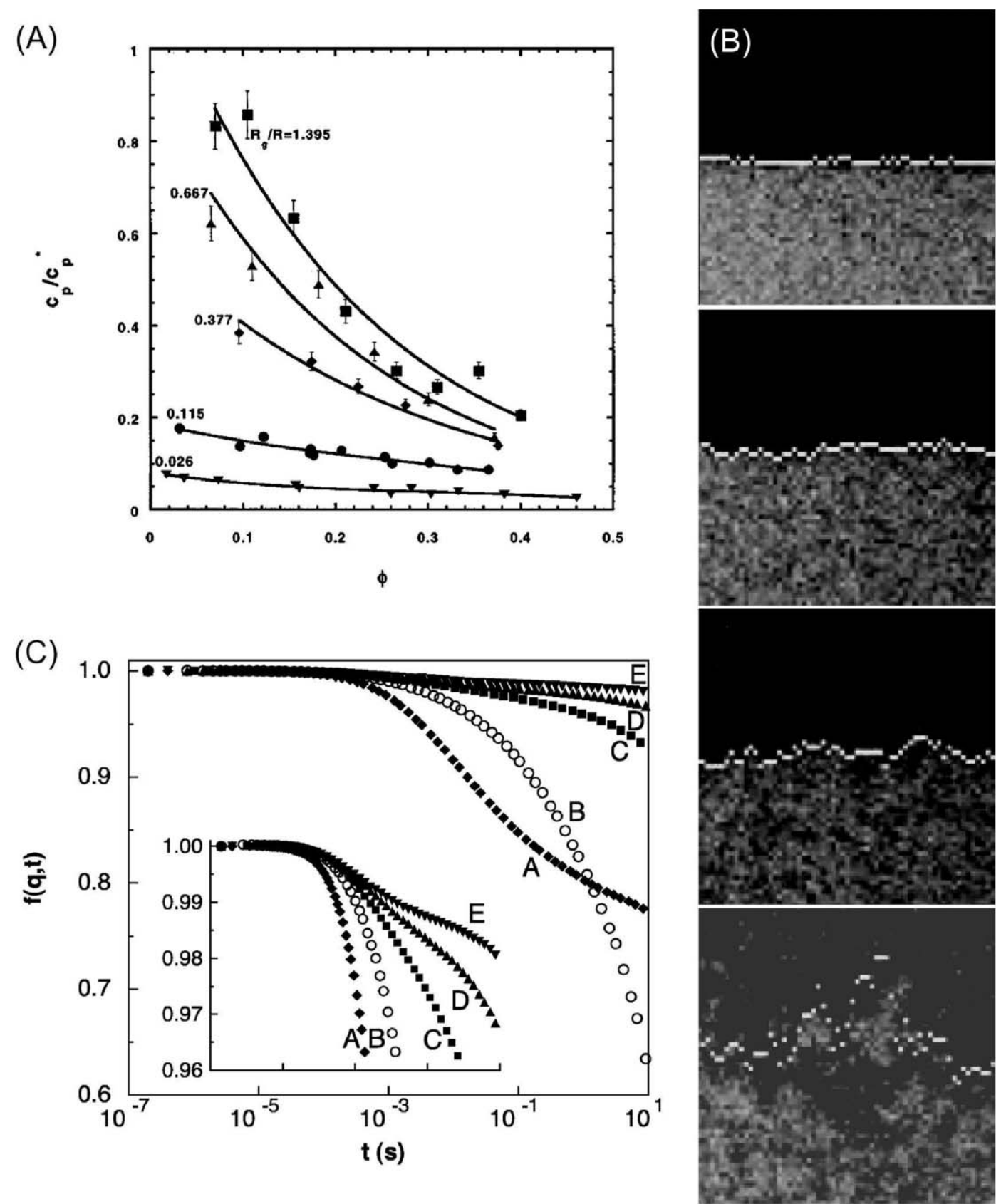

Fig. 2 Entropic effects in colloid-polymer mixtures. (A) Phase separation observed in the polymer concentration $\left(c_{\mathrm{p}} / c_{\mathrm{p}}{ }^{*}\right)$-colloid packing fraction $(\phi)$ phase diagram. As a function of the polymer-colloid size ratio $R_{\mathrm{g}} / R$, fluid-gel $\left(R_{\mathrm{g}} / R=0.026\right)$, fluid-solid $\left(R_{\mathrm{g}} / R=0.115\right)$, and fluid-fluid $\left(R_{\mathrm{g}} / R=0.377-1.395\right)$ phase boundaries are observed. ${ }^{67}$ Reused with permission from S. Ramakrishnan, M. Fuchs, K. S. Schweizer, and C. F Zukoski, Journal of Chemical Physics, 116, 2201 (2002). Copyright 2002, American Institute of Physics. (B) Capillary waves at a colloidal fluid coexistence on approaching the gas-liquid critical point. ${ }^{70}$ The gas-liquid interface gets rougher on approaching (top to bottom) the critical point from the two-phase region. From ref. 70. Reprinted with permission from AAAS. (C) A plateau after the inflection point in the time-dependence of the dynamic structure factors represents glassy behaviour. Glass phase in the presence of repulsive interactions (sample A) disappears when attractive interactions are added, but reappears for stronger attractive interactions (samples D and E) $;{ }^{71}$ the inset shows an expanded region of the same data that highlights this second plateau. From ref. 71. Reprinted with permission from AAAS.

We only discuss here the simplest electrostatic interactions. We do not discuss, for example, the van der Waals ("dispersion") interaction in colloids, which is a shortranged $(0-10 \mathrm{~nm})$ attraction that can be strong $\left(1-100 k_{\mathrm{B}} T\right.$; ref. 16, chapter 5). It is the primary cause of uncontrolled aggregation in colloidal systems, and most colloids are "stabilized" either sterically (usually short chain-like molecules attached to the colloid, making its surface act like a toothbrush) or by surface charge groups to prevent particles from approaching each other close enough in order to aggregate. Note that such stabilization itself implies at least a short-ranged repulsion. The dispersion interaction can be minimized by matching particle and solvent refractive index. 
Colloidal suspensions of highly charged spheres in a solvent with counter ions interact via a screened Coulomb interaction and form low density crystals (Fig. 3A). Molecular dynamics simulations ${ }^{88-91}$ have studied the phase behaviour of particles interacting through a screened Coulomb (Yukawa) potential (Fig. 3B). The Yukawa pair potential has the form

$$
U(r) \approx \lambda \frac{\exp (-\kappa r)}{r}
$$

where $1 / \kappa$ is the Debye-Hückel screening length (see Table 1 ). This system exhibits a fluid phase as well as face-centred cubic
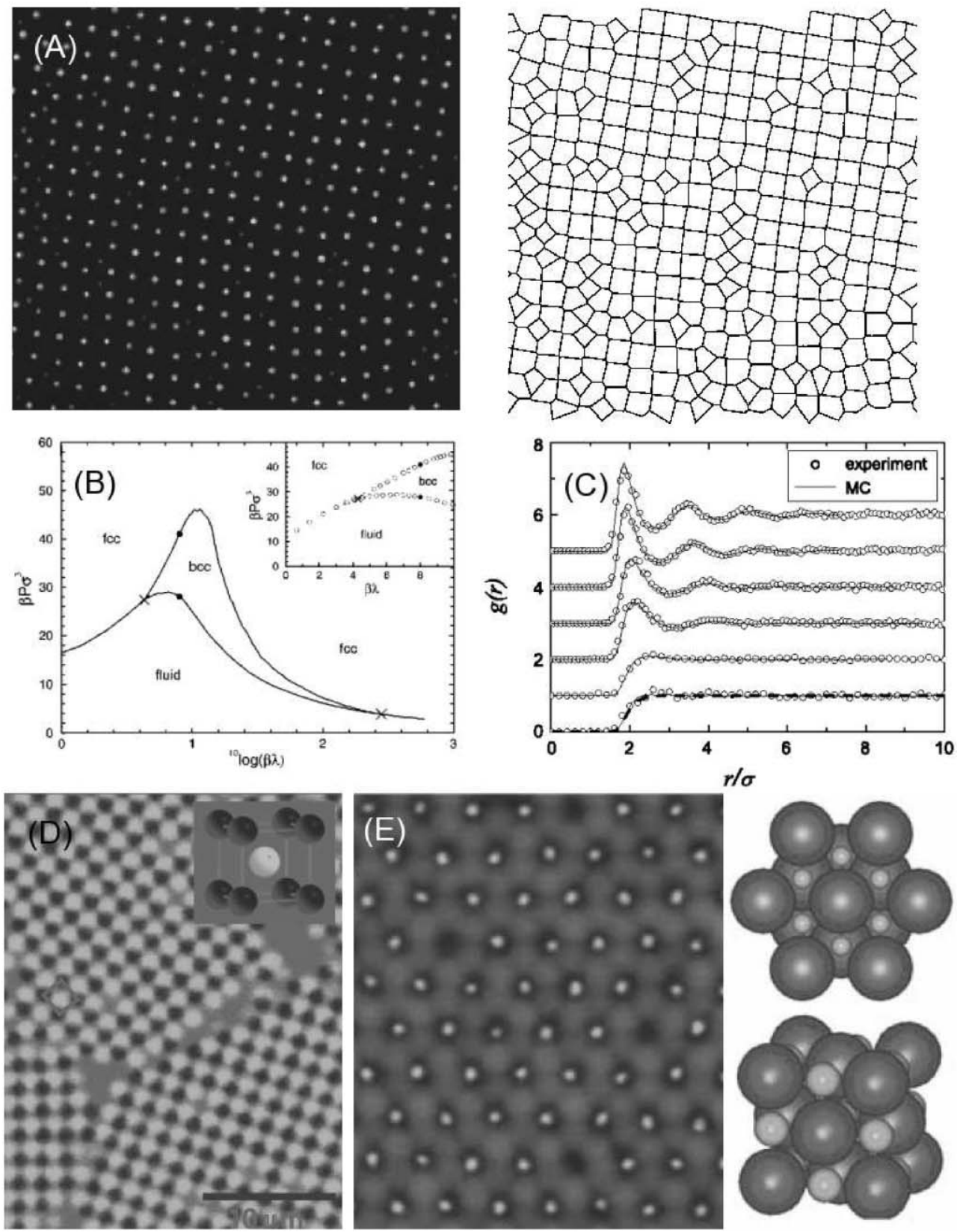

Fig. 3 Charged colloids. (A) Two-dimensional snapshot of low density colloidal crystal and its Voronoi construction. ${ }^{5}$ The softness of the crystal is exemplified by the bending of the crystal planes. Reused with permission from Jessica A. Weiss, David W. Oxtoby, David G. Grier, and Cherry A. Murray, Journal of Chemical Physics, 103, 1180 (1995). Copyright 1995, American Institute of Physics. (B) Phase diagram (from ref. 91) of the hard-core Yukawa system shows fluid, bcc, and fcc phases as a function of $\beta \lambda \equiv \lambda / k_{\mathrm{B}} T$ and a density/pressure variable. Reused with permission from Evert Jan Meijer and Fouad El Azhar, Journal of Chemical Physics, 106, 4678 (1997). Copyright 1997, American Institute of Physics. (C) One can extract the pair correlation function $g(r)$ experimentally and fit to ones obtained from simulation by varying model parameters. ${ }^{95}$ (D, E) Ionic colloidal crystals composed of opposite-charged spheres of different size-ratios. Shown here are (left ${ }^{15}$ a CsCl crystal of nearly equal-sized spheres and an LS-type crystal (right) ${ }^{14}$ with highly asymmetric size ratio. D reprinted with permission from ref. 15. Copyright (2005) by the American Physical Society. http://link.aps.org/abstract/PRL/v95/e128302. E reprinted with permission from ref. 14, copyright (2005) Nature Publishing Group. 
(fcc) and body-centred cubic (bcc) solid phases as a function of $\lambda / k_{\mathrm{B}} T$, with fluid-bcc, fluid-fcc, and bcc-fcc coexistence regions, as well as a fluid-bcc-fcc triple point.

This system allowed a beautiful test of universal criteria for the melting of solids. The Hansen-Verlet rule stipulates that the first peak of the structure factor $S(k)$ [the Fourier transform of the pair correlation function $g(r)$ ] reaches a constant value at the melting temperature. The value of $S(k)$ for $\kappa a=1.6$ (soft spheres) to $\kappa a=14.3$ (nearly hard spheres) ranged from 2.8 to 3 (in comparison with the value of $2.85^{92}$ ). The Lindemann criterion for melting ${ }^{93}$ uses the rms displacement as a fraction of the lattice spacing $\sqrt{\delta u^{2} / a^{2}}$. In the context of this model, melting was seen to occur at $\sqrt{\delta u^{2} / a^{2}}=0.19$.

Now to experiment. Control of electrostatic interactions is achieved via the Debye-Hückel screening length (Table 1), which in turn is controlled via the solvent salt concentration and/or ion-exchange resins. The existence of bcc and fcc phases, as well as the predicted phase sequences, have been experimentally established as well. ${ }^{19,94}$ Microscopy has been used to obtain quantitative information about crystals via real-space analysis of particle positions. Shown in Fig. 3A is a low-density colloidal crystal and its Voronoi construction. ${ }^{5}$ Pair correlation functions can be obtained via three-dimensional confocal microscopy and compared directly to those obtained from computer simulations (Fig. 3C). ${ }^{95}$ In experiments on highly-charged colloids, the presence of surfaces (especially in microscopy where the proximity of the cover-glass is always an issue), and the need to characterize particle charge, makes quantitative comparisons of different experiments especially challenging. In nonaqueous suspensions of highly charged colloids, fluid, bec and fcc as well as rhcp phases were observed. ${ }^{7}$ In a similar system, re-entrant melting and an rhcp crystal structure was also observed. $^{95}$

Effective attractions have been reported between colloids with like charges. ${ }^{96-98}$ The issue has been clouded experimentally by several issues which have been dealt with one by one: apparent attractions due to structure factor inversion and imaging artifacts, ${ }^{99,100}$ hydrodynamic effects ${ }^{101}$ and surface effects. $^{102}$ Careful characterization of particle charge and solvent conductivity, ${ }^{103}$ and control of the electric potential at the proximate surfaces, ${ }^{7}$ are therefore warranted. Nevertheless, there are issues remaining to be resolved. The mean-field theory for charged colloids is the DLVO theory $\left(\mathrm{see}^{104}\right)$, which predicts a purely repulsive pair potential. One could ask whether an effective attraction in the pair potential could arise from many-body effects. The effect of a third body on effective pair interactions has been considered explicitly in an optical tweezer experiment coupled with numerical simulations. ${ }^{105}$ Two particles were confined to a line and a third to a point at variable distance $d$ from the line. The total potential of all three particles was found to be significantly dependent on the distance $d$ and less than the sum of the pairwise terms. That is, for small $d$, the three-body term was negative and of order $20 k_{\mathrm{B}} T$. Thus many-body effects can lead to effective attractions. Indeed, the importance of higher order terms increases with colloid concentration and the reduction of the pairwise interaction can be physically attributed to the additional charged particles effectively blocking the mutual pair interactionthis is termed macro-ion screening, ${ }^{106}$ in analogy with DebyeHückel screening. As noted in ref. 107, experiments ${ }^{19,94,108}$ have in common a broad fluid-solid or solid-solid coexistence in the low-salt regime, with large density jumps between phases $\left(200 \%\right.$ at fluid-solid coexistence in $\left.{ }^{94}\right)$. This is anomalous. The fluid-solid density jump for hard spheres is $10 \%$, and the soft, long-range, repulsive interactions of the meanfield theory give rise to very narrow coexistence regions, and density jumps should, in fact, decrease as the interaction becomes softer.

The phase behaviour at high density of binary colloids of similar size but opposite charge was reported. ${ }^{15,14}$ Bartlett et $a l .{ }^{15}$ varied the potential between the spheres from $+5 k_{\mathrm{B}} T$ (repulsive) to $-3 k_{\mathrm{B}} T$ (attractive) for equal size spheres. Charge inversion was achieved by leaving a ferromagnetic wire in the suspensions as a catalyst to generate free $\mathrm{Br}^{-}$ions, and taking advantage of the fact that this process occurs gradually, as a function of time, and is quantitatively different for colloids synthesized in different batches. Leunissen et al. ${ }^{14}$ varied the size ratio between the positive and negatively charged spheres. In this case, the charge inversion is achieved using the complicated behaviour of the tetrabutylammonium bromide salt. In both cases, the $\mathrm{Br}^{-}$ion is implicated, and the mechanism is likely as follows. The solvent partially dissociates giving rise to $\mathrm{HBr}$ - the proton then most likely associates with the steric stabilizer on the spheres, giving them a positive charge. The charge inversion then is in fact a suppression of this mechanism via a suppression of the dissociation of the bromocyclohexane solvent. The remarkable feature is that the attractive interaction is weak enough for thermodynamicallyreversible equilibrium structure formation, as opposed to irreversible aggregation. This extends the thermodynamic analogy for colloids from "atomic" to "molecular" systems, and is thus an important result. Ionic crystals with facecentered cubic, caesium chloride, and sodium chloride structures were observed as well as $L S$ and $L S_{6}$ structures for larger size ratios. Fig. $3 \mathrm{D}^{15}$ and $\mathrm{E}^{14}$ show confocal micrographs and unit cells of the $\mathrm{CsCl}$ and LS type binary crystals.

Electrostatic interactions have been used ${ }^{109}$ in binary (equalsized, but chemically dissimilar) nanoparticle metal colloids to realize the diamond lattice structure. This structure is soughtafter because it can be used to make photonic materials with a complete three-dimensional photonic bandgap (ref. 110, see ref. 111 for a progress report on diamond-structured photonic crystals).

\section{Tunable interactions}

While the control of particle shape, size, volume fraction, charge and solvent screening lengths are important control parameters for colloid experiments, none of them affords active control. Methods for active control have been explored by the colloid community (see for example ref. 112, 113) and are discussed in detail below. As it happens, active control also introduces anisotropy to the interactions. 


\subsection{Spatially-uniform electromagnetic fields}

Colloids experience an averaged dipolar interaction in the presence of either high-frequency alternating electric fields or static magnetic fields. Dipolar colloids have been extensively studied for their potential as "electro-rheological"114 and "magneto-rheological" fluids ${ }^{115}$ with a field-switchable yield stress. Low-frequency electric fields are often more complicated due to fascinating electrohydrodynamic effects. ${ }^{116}$

3.1.1 Ac electric fields: control of dipolar interactions. Colloidal suspensions are composed of particles in a solvent, where particle and solvent usually have different dielectric constants. In electric fields of strength $\approx 0.1-1 \mathrm{kV} \mathrm{mm}^{-1}$, chain-like or columnar structures form that result in concurrent changes in the macroscopic rheological properties - the "electro-rheological (ER) effect",30,31 (see ref. 114 for a survey of the field). Particle chaining (which is a one-dimensional close packing along the electric field direction) can be understood by modelling the sphere-sphere interaction as an induced dipole-dipole interaction where the dipoles point along the field direction. ${ }^{117}$

The simplest systems to study are colloids in a sinusoidallyalternating field. At mega-Hertz frequencies, particles see an average field and effects of ion migration are minimized. The point-dipole approximation assumes that the dipole induced on a sphere by the applied external field $E_{0}$ is not affected by neighbouring spheres-this is true when the sphere-sphere distance is much greater than the particle radius, i.e. $R \gg a$. The dipolar interaction energy $U(R)$ between two spheres separated by displacement vector $\vec{R} \equiv(R, \theta, \phi)$ in this limit is

$$
U_{\text {dip }}(R, \theta)=-\frac{4 \pi \varepsilon_{0} \varepsilon_{\mathrm{f}} \beta^{2} a^{6} E_{0}^{2}}{R^{3}}\left[\frac{3 \cos ^{2} \theta-1}{2}\right]
$$

where $\beta \equiv \frac{\varepsilon_{\mathrm{p}}-\varepsilon_{\mathrm{f}}}{\varepsilon_{\mathrm{p}}+2 \varepsilon_{\mathrm{f}}}$, and $\varepsilon_{\mathrm{p}}$ and $\varepsilon_{\mathrm{f}}$ are particle and fluid dielectric constant. The salient characteristics of this interaction are its angular dependence (it switches sign at $\theta \approx 54.7^{\circ}$ ), its long-range character and its sharp particle size dependence.

The dynamics of structure formation is interesting. Chain formation (Fig. 4A) is followed by a coarsening of the chains $^{118}$ into sheets. Eventually, the sheets transform into an equilibrium three-dimensional crystal structure that is not close-packed but is a body-centred tetragonal ordering composed of chains. ${ }^{6,119-121}$ Fig. 4B shows a view ${ }^{122}$ along the direction of the field - the structure in the field direction is that of continuous chains. It has recently been reported ${ }^{123}$ that dipolar spheres exhibit a cellular pattern after the chaincolumn formation (Fig. 4C) when other competing interactions are suppressed. These new structures, reported for dipolar spheres in the non-Brownian regime $(\lambda \gg 1)$, demonstrate that careful control of interactions can yield surprises even in well-studied model systems.

The point-dipole approximation is, in general, not valid. Indeed, the most interesting recent development are nanoparticle colloids with a surface coating that enhance the ER effect ${ }^{124,125}$ (Fig. 4D). Here the observed yield strength ( $\approx 130 \mathrm{kPa})$ is 20 times what one would expect given linear dielectric and conductive responses of the colloidal suspension.
Finally, electric fields can be used to induce martensitic solid-solid transitions between close packed and tetragonal crystals $^{6,126}$ (Fig. 4E).

3.1.2 Magnetic fields. Mechanisms of structure formation in mega-Hertz ac electric fields are close to those in magnetic fields. Colloids in magnetic fields show the magnetic analog of the ER effect - the "magneto-rheological (MR) effect". Indeed from the point of view of commercial applications, MR fluids are currently more promising. ${ }^{127}$ The main difference experimentally is that magnetic materials are generally opaque and therefore optical studies in this case are limited to two dimensions.

Very beautiful two-dimensional studies in magnetic colloids have been carried out. The KTHNY mechanism ${ }^{128,129}$ predicts a two-stage melting transition in two-dimensional crystals where the solid and liquid phase are separated by an intermediate hexatic phase. Zahn et al. demonstrated ${ }^{4}$ that a two-dimensional system of interaction dipolar colloids does indeed exhibit a two-stage melting transition as the effective temperature $\Gamma^{-1}$ (see Table 1) is raised : the crystal-hexatic phase is mediated by the unbinding of dislocation pairs, and the hexatic-liquid transition by the unbinding of diclination pairs. Snapshots of these phases ${ }^{130}$ are shown in Fig. 4F. Melting occurs when a modified Lindemann melting parameter $\gamma_{\mathrm{L}}=\left\langle\left(\Delta u_{\mathrm{j}}(t)-\Delta u_{\mathrm{j}+1}(t)\right)^{2}\right\rangle \approx 0.03$.

Fluid-fluid phase coexistence has been predicted in systems of binary dipolar spheres composed of two species of spheres with differing dipole moments. ${ }^{131}$

3.1.3 Low frequency ac and de fields: electrohydrodynamics and electrokinetics. Low-frequency electric fields have interesting and complicated effects when applied to colloidal suspensions. Low frequency electric fields (from a few $\mathrm{Hz}$ to a few $\mathrm{kHz}$ ) cannot be treated as an average interaction. In particular, there are two effects that make low-frequency fields complicated. First, at low frequencies, charge migration timescales are comparable to the timescale of one period of oscillation, and the full complex response must be taken into account. Indeed low-amplitude electric fields are used in impedance spectroscopy of colloids ${ }^{132}$ to yield important insights into colloidal suspension properties. Second, a colloidal particle must move less than a small fraction of its diameter in one period of the alternating field in order for the particle to see a period averaged field. If this is not satisfied, rich low-frequency electrohydrodynamic phenomena can be observed, giving rise to dynamical stationary states (circulating bands of particles ${ }^{116}$ as well as surface crystallization $\left.^{133,134}\right)$.

While the thermodynamic analogy is powerful, it should be noted that external fields do provide energy inputs to the system, and the difference between equilibrium thermodynamics and a non-equilibrium steady state can experimentally be a subtle matter. Indeed non-equilibrium behaviour is essential for "active" granular systems ${ }^{135}$ and the crossover from the granular to the colloidal regime (characterized for example by the Peclet number or the gravitational height, see Table 1) is a matter of great interest. 

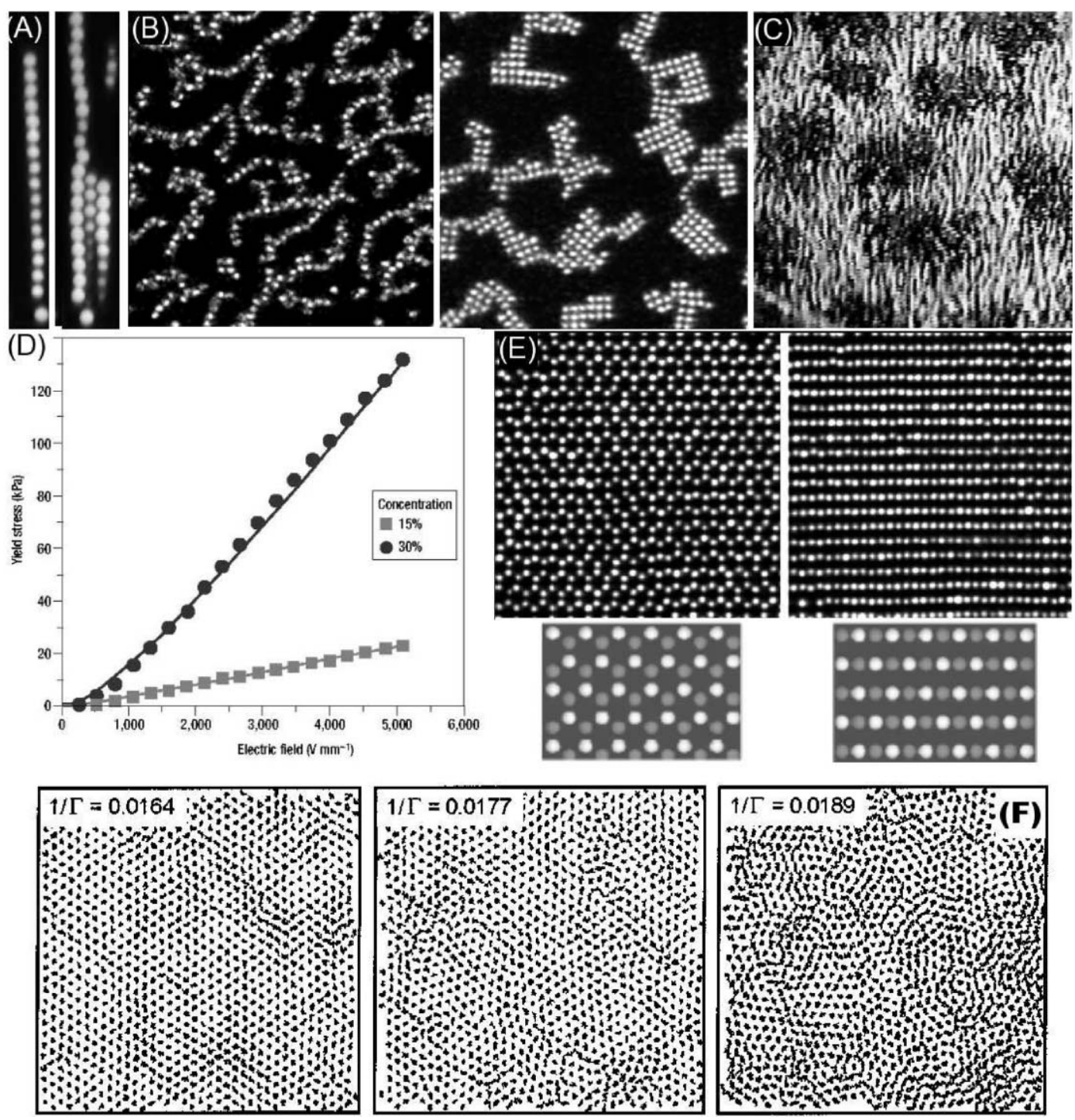

Fig. 4 (A) Chain formation (left) in an vertical ac electric field. Chains coarsen into sheets (right). ${ }^{122}$ (B) Two snapshots in the time evolution of the formation of BCT crystals (chains-sheets-BCT) in dipolar colloids. ${ }^{121}$ Electric field points into the page. Reprinted with permission from ref. 121, copyright (2002) World Scientific Publishing Co. (C) Cellular structures in $87 \mu \mathrm{m}$ spheres in an external electric field. ${ }^{123}$ All fields above are $\approx 1-2 \mathrm{kV} \mathrm{mm}{ }^{-1} \mathrm{rms}$. Reprinted with permission from ref. 123. Copyright (2005) by the American Physical Society. http://link.aps.org/abstract/ PRL/v95/e258301. (D) Giant electro-rheological effect in nanoparticle colloidal suspensions: ${ }^{124}$ the yield strength is 20 times the predicted value. Reprinted with permission from ref. 124, copyright (2003) Nature Publishing Group. (E) Electric field driven martensitic transition from a closepacked (left) to a tetragonal (right) crystal: confocal micrographs (above) and model (below) of two-layer projections of hexagonal packed layers. ${ }^{126}$ The in-plane order remains unchanged but the stacking of layers changes with increasing field $\left(\approx 0.1 \mathrm{kV} \mathrm{mm}{ }^{-1} \mathrm{rms}\right)$. Reprinted in part with permission from ref. 126, copyright (2004) by the American Physical Society. http://link.aps.org/abstract/PRL/v92/e058301. (F) Real-space structure of colloidal crystal, hexatic and liquid phases ${ }^{130}$ in two-dimensional samples of superparamagnetic colloidal particles where the magnetic dipolar interaction (characterized by $\Gamma$, see Table 1) is repulsive and tunable via an external magnetic field. The hexatic phase displays dislocations while the liquid phase displays disclinations. Reprinted with permission from ref. 130, copyright (2000) by the American Physical Society. http:// link.aps.org/abstract/PRL/v85/p3656.

A DC electric field coupled to a feedback loop that receives input from video microscopy has been demonstrated to trap individual particles from the micron-scale to nanoparticles. ${ }^{136}$ While this technique, which is shown impressively to freeze out the Brownian motion of single particles) has scarcely been used in colloidal systems, it shows great promise for single-particle trapping in the singlemolecule regime where optical tweezers (discussed next) would fail.

\subsection{Non-uniform electromagnetic fields}

While nonuniformities are not desirable in situations designed for uniform electric fields, they have some unique advantages. First, field gradients exert directional forces on colloidal particles. Such non-uniform fields are of widespread interest for cell separations in biotechnology, ${ }^{137}$ since many biological systems are colloidal. Second, field gradients can be used to map out phase diagrams with fields used either as surrogate 
thermodynamic variables or as a means to create density gradients.

3.2.1 Optical tweezers. A focused laser beam can be used to create spatial optical field gradients which in turn can be used to trap and measure forces in colloids and biomolecules (hence "optical tweezers"). ${ }^{138-140}$ Modifications of the technique have allowed time-varying optical traps at ${ }^{141-144}$ rates that are comparable to natural time scales for the dynamics of micronscale colloids, as well as simultaneous trapping and control of two-photon fluorescence in colloidal microspheres using a femtosecond laser. ${ }^{145}$ Simultaneous trapping and imaging in three-dimensions has been demonstrated in concentrated colloidal suspensions. ${ }^{146}$ Laser tweezers enable the study of rheology in colloidal suspensions and other complex fluids (see ref. 147 for a review).

These technical developments have enabled the study of colloids in the presence of confining fields. ${ }^{148}$ Single-file diffusion has been observed in colloids confined to narrow channels. ${ }^{149}$ Direct measurement of three-body interactions (referred to earlier) has been carried out in a system where two particles are confined to an optical line trap ${ }^{105}$ and a third to a point trap. The phase behaviour of two-dimensional suspensions in periodic light fields was probed via optical microscopy_laser-induced freezing and melting was observed. ${ }^{150}$ Such light fields can be used to mimic periodic surfaces and are therefore of great interest.
Optical vortices can be used to confine colloidal microspheres to one-dimensional rings. ${ }^{151}$ Particles so-trapped still exhibit Einstein-like diffusivity-however, the value of the effective diffusion coefficient is more than 100 times the value of a freely diffusing colloidal sphere. Optical tweezers have been used ${ }^{152}$ to create defects in crystals as a means to study defect dynamics.

Optical tweezer arrays can be used to control colloidal crystal growth. ${ }^{146}$ Holographic tweezer arrays have been utilized to make novel quasi-crystalline structures in two and three dimensions, as well as to engineer defects in such structures (Fig. 5A). ${ }^{153}$

3.2.2 Dielectrophoresis. Dielectrophoresis is used extensively in colloidal separations in biology and medicine. It holds much promise for obtaining monodisperse colloids from polydisperse suspensions by separating out large and small particles. Indeed, the procedure has been reported successfully for different species of carbon nanotubes. ${ }^{154}$

It has also been used to establish controlled concentration gradients in order to determine colloidal equations of state in one sample. The idea of determination of equations of state was first achieved using gravity to create a concentration gradient. ${ }^{28}$ However, colloidal phase behaviour dynamics is strongly affected by gravity. A new dielectrophoresis technique for achieving volume fraction gradients ${ }^{155}$ has the advantage that the densifying force can be turned off at will, or left on
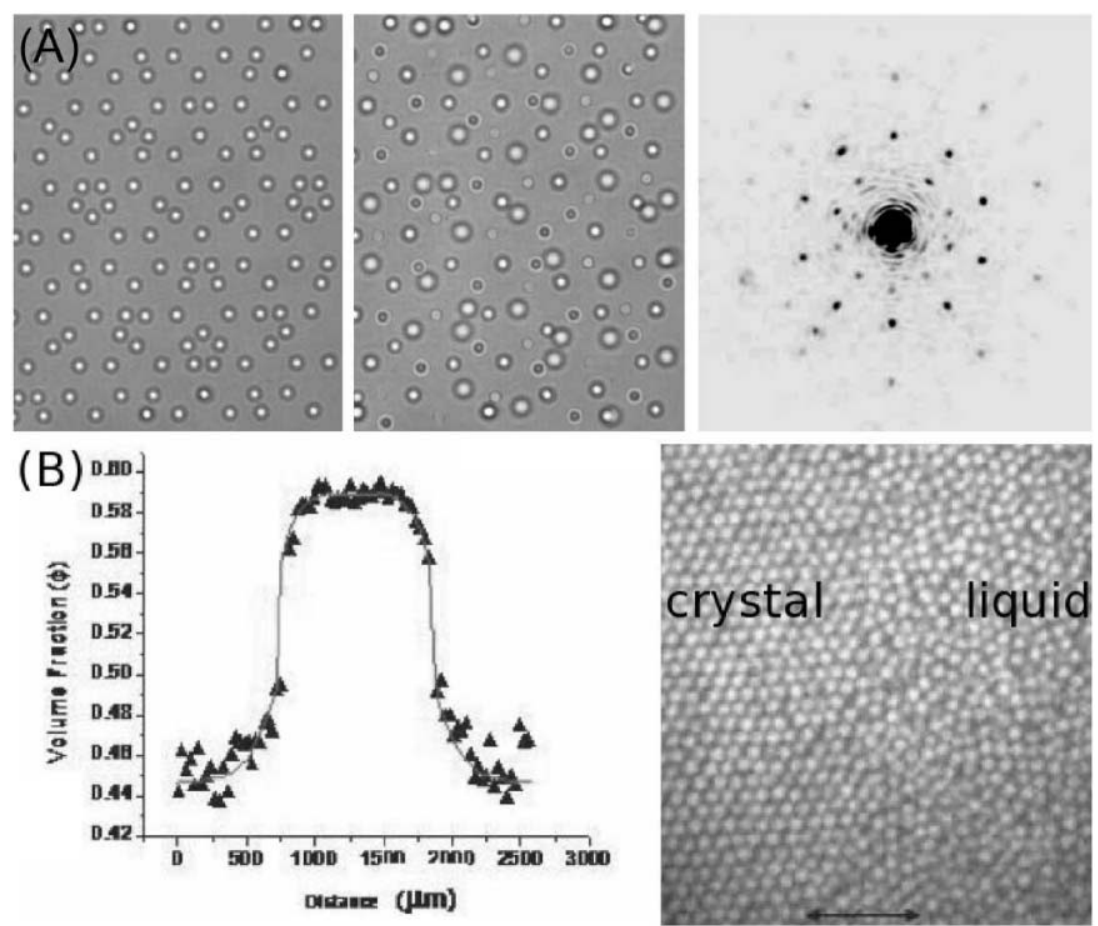

Fig. 5 (A) Holographic optical tweezer arrays can be used to extend the study of colloidal crystals to two- and three-dimensional quasicrystalline (QC) structures. ${ }^{153}$ Shown are colloidal particles trapped in a 2D projection of a 3D icosahedral QC lattice (left), particles displaced into the full 3D configuration (middle) and an optical diffraction pattern showing 10-fold symmetric diffraction peaks (right). (B) The colloid volume (packing) fraction $\phi$ is an important control parameter in studies of structure formation. Dielectrophoretic forces have been used ${ }^{155}$ to create volume fraction gradients (left) to map out the entire hard-sphere phase diagram in one sample-shown (right) is a crystal-liquid interface in such a sample. Reprinted with permission from ref. 155, copyright (2006) by the American Physical Society. http://link.aps.org/abstract/PRL/v96/e015703. 
provided that the (electric dipolar-thermal) $\Lambda$ parameter (see Table 1) is much less than one. Demonstrated (Fig. 5B) for hard spheres, this technique is generalizable for spheres interacting with other pair potentials, and is thus very powerful.

Dielectrophoresis, ${ }^{156}$ in addition to ac electrophoresis techniques, ${ }^{157}$ has been used as a viable means to concentrate nanoparticles suspensions as well as control their assembly. ${ }^{156}$

\subsection{Other external fields: shear and anisotropic solvents}

3.3.1 Shear. Shear can have different effects on a colloidal crystal. ${ }^{158}$ First, a crystal can only be sheared without being completely destroyed ("melted") if the shear gradient direction is perpendicular to an "easy" plane - e.g. the (110) and (111) planes in a bcc and fcc crystal, respectively. Second, even when sheared along an easy plane, disordering occurs at high enough shear amplitudes. Finally, nucleation and growth rates (discussed in section 4) depend on the difference in chemical potential between crystal and fluid phases. The "effective" chemical potential difference is affected by flow. The melting of a body-centred cubic (bcc) crystal was observed first via light scattering. ${ }^{159}$ In a two-stage melting process, the crystal melted at low shear rates into two-dimensional hexagonal close packed (hcp) planes that freely slipped across each other, then melting at higher shear rates (estimated to be $\approx 10 \mathrm{~Hz}$ ) completely into an amorphous structure with string-like correlations.

Hard sphere fluids crystallize with or without shear as one increases particle concentration. ${ }^{160}$ However, in the presence of shear there is a non-equilbrium re-entrant fluid phase at high concentration, with the value of this threshold concentration decreasing with increasing shear rate, until the crystal phase completely disappears at a high-enough shear rate.

In charged colloids at low particle packings, a similar transition is observed with the important exception that the structural rearrangements are continuous and not abrupt. ${ }^{161}$ At higher particle packings, the effect of parallel-plate shear has been studied in a confined suspension of charged colloids. $^{162}$

The effect of the geometric confinement is to create a new ordered structure where particle layers buckle in such a way that the configurations observed optimize packing in a plane that includes the $z$ direction perpendicular to the shear direction and an axis that (with reference to the unsheared hexagonal plane) is along one of the touching-particle directions $(\pi / 3)$ from the shear direction.

Shear can have dramatic effects on the stability of colloidal suspensions. ${ }^{163}$ Guery et al. demonstrated that shear can induce irreversible aggregation in a dilute suspension of large ( $5 \mu \mathrm{m}$ and therefore non-Brownian, $\phi=0.1$ ) solid droplets that are stable in the absence of shear. Indeed the time to aggregation was seen to decrease exponentially with the shear rate.

A proviso is in order. Unlike other interactions discussed in this paper, shear cannot, even in principle, be considered as a thermodynamic variable, as it has been shown ${ }^{164,165}$ that a crystal-liquid coexistence in the presence of shear cannot be accounted for by invoking a non-equilibrium analog of a chemical potential. Physically, shear is directly involved in transporting particles within and between phases.

More complex forms of shear are realized in the process of spin-coating liquids. A recent study has probed the interesting and complicated phenomenon of colloidal crystallization while the suspension is spincoated onto a substrate. ${ }^{166}$ Here the angular velocity of the spinner can control the thickness as well as crystal quality of the resulting colloidal sediment.

3.3.2 Colloids in liquid crystals. Liquid crystals exhibit orientational anisotropy, and the inclusion of a micron-sized colloidal particle in a nematic liquid crystal (which has orientational order that is often described by a "director" field) immediately introduces a great deal of complexity. First, liquid crystal molecules "anchor" to a surface at a given orientation: thus the shape of the colloidal particle introduces a defect that gives rise to characteristic long-range textures in the liquid-crystal director field. Introducing a second particle induces a similar director field around the second particle, and thus the two particles interact via liquid-crystal anisotropy. The interaction of colloidal particles in the presence of such an anisotropic medium has indeed been studied experimentally and theoretically ${ }^{167-175}$ (see ref. 170 for a review). A colloidliquid-crystal mixture phase separates into colloid-rich and colloid-poor regions, with the phase separation depending sensitively on the liquid crystal anchoring conditions at the colloid surface.

Polystyrene and PMMA microspheres in a lyotropic liquidcrystalline medium have also been studied. ${ }^{176}$ The colloids do not appear to significantly modify the phase diagram of the lyotropic systems and were found to be encapsulated within multilamellar vesicles.

\subsection{Increasing complexity}

One is often faced with the possibility (or necessity) to deal with multiple interactions in one system. The control of more than one kind of interaction, not surprisingly, can increase both the complexity and the degree of control over phase behaviour. A few applications of multiple colloidal interactions are discussed briefly below.

Yethiraj and van Blaaderen ${ }^{7}$ demonstrated (Fig. 6A) that charged colloids in a solvent with a long Debye-Hückel screening length with an added field-induced dipolar interparticle interaction produces a rich phase sequence that includes (as a function of field strength and packing fraction) body-centred cubic (bcc), face-centred cubic (fcc), bodycentred tetragonal (bct) and body-centred orthorhombic (bco) phases, as well as a novel phase that is fluid-like in two dimensions and solid-like along the direction of the external field. An important upshot of this phase diagram is the ability to change phase with electric field as a (reversible) control parameter.

Manoharan et al. ${ }^{13}$ demonstrated (Fig. 6B) that clusters of microspheres can be created of regular and controllable size by preparing them in an emulsion droplet and then drying. The authors explain the regularity of the clusters by arguing that they minimize the second moment of the mass distribution. 


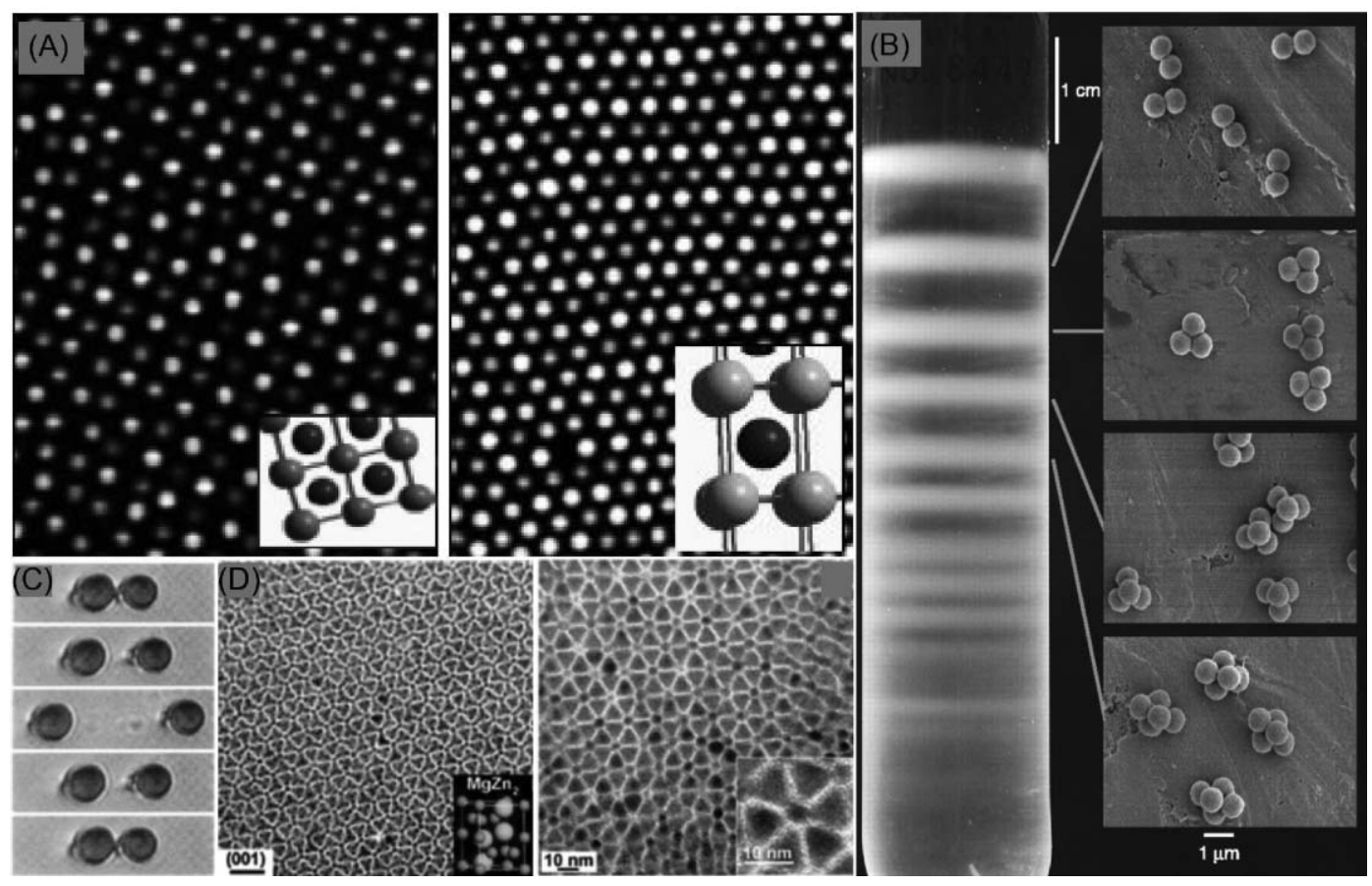

Fig. 6 Multiple interactions. (A) The combination of electrostatic repulsion and the anisotropic dipolar interaction gives rise to a rich phase diagram that includes space-filling body-centred tetragonal (bct, left) body-centred orthorhombic (bco, middle) crystals (the field points out of the page) at volume fractions near 20\%. ${ }^{7}$ Reprinted with permission from ref. 7, copyright (2003) Nature Publishing Group. (B) Colloidal polystyrene microspheres dispersed in an emulsion droplet form "small colloidal molecules" (right) of different sizes. ${ }^{13}$ Different cluster sizes are separated from each other (left) by centrifugation in a density gradient. From ref. 13. Reprinted with permission from AAAS. (C) The effect of two anisotropic interactions: colloids in a magnetic field and a liquid-crystal environment where the field strength (ramped up and then down) controls the colloid separation. ${ }^{177}$ Reprinted with permission from ref. 177, copyright (1997) by the American Physical Society. http://link.aps.org/abstract/PRL/v79/ p4862. (D) Two examples of structures seen in binary nanoparticles superlattices: ${ }^{181}$ TEM images of (left) triangular LaF $F_{3}$ nanoplates and gold nanoparticles; (right) $6.2 \mathrm{~nm} \mathrm{PbSe} / 6 \mathrm{~nm}$ Pd nanospheres form a $\mathrm{MgZn}_{2}$ lattice. Here, electrostatic and dipolar and van der Waals interactions as well as the capillary forces at the gas-liquid interface are at play. Reprinted with permission from ref. 181, copyright (2006) Nature Publishing Group.

Different sizes are beautifully separable via centrifugation in a density gradient.

Colloidal forces have been measured by Poulin et al. in colloidal ferrofluid droplets in the presence of a magnetic field (Fig. 6C) - the repulsion in the presence of a magnetic field competes with the attraction due to the anisotropic liquid crystal environment. ${ }^{177}$

Lowen et al. demonstrated ${ }^{178}$ that shear can be used effectively to control crystal orientation in thin colloidal layers that are crystalline due to magnetic dipolar interactions.

Lettinga et al. ${ }^{179}$ studied the effect of shear on the gas-liquid critical point in a colloid-polymer mixture. The polymer induces the attractions that give rise to a fluid-fluid transition in the first place, and the distance from the critical point can be controlled. Shear flow also suppresses the capillary waves at a depletion-induced gas-liquid transition. ${ }^{180}$

Shevchenko et al. ${ }^{181}$ demonstrated a startling array of structures (two examples in Fig. 6D) in binary nanoparticle superlattices. Numerous lattice structures are realized by utilizing nanoparticles of different sizes and shapes, as well modifying the ionic environment. Crystals are prepared by evaporative drying. Electrostatic and dipolar and van der Waals interactions are at play, and the phenomena are apparently not completely dominated by surface tension at the gas-liquid interface during evaporative drying. This is surprising, as one expects the capillary forces experienced by colloids immersed in a liquid on a solid substrate to be much larger than the thermal energy $k_{\mathrm{B}} T{ }^{182}$

Leunissen et al. ${ }^{14}$ have reported experimental observation of the phenomenon of lane formation ${ }^{183}$ in binary oppositecharged colloids in the presence of an imposed electric field.

The effect of electric fields in colloid-liquid-crystal mixtures ${ }^{170}$ has been studied. The nature of the electric dipolar interaction was not significantly different from the nematicdefect driven dipolar interaction, and all that was observed was a change in the colloid chain spacing.

The interplay between gravity and electric field in colloidal crystallization $^{126}$ gives rise to a layer-by-layer martensitic transition (see Fig. 4D) from a gravitationally-induced closepacked crystal at zero fields to a less dense tetragonal (bct) crystal on increasing the electric field beyond a threshold that depended on depth in the sediment.

\section{Crystal nucleation and growth}

Tunability - and the concomitant possibility of rapidly cycling between thermodynamic phases - is perhaps of most value in the study of events that are difficult to study, either because they are very rapid, very rare or extremely slow. Two fundamental problems that fall into this category are crystal 
nucleation and the glass transition. In this section, the current status in the problem of crystal nucleation and growth is discussed.

\subsection{Crystal nucleation}

Crystal nucleation has been studied extensively, yet the rate of crystal nucleation is exceedingly difficult to predict. According to classical nucleation theory, the total free energy cost to form a spherical crystallite of radius $R$ is given by

$$
\Delta G=-\frac{4 \pi}{3} R^{3} n_{\mathrm{s}}|\Delta \mu|+4 \pi R^{2} \gamma
$$

where $|\Delta \mu|$ is the chemical potential difference between the solid and liquid, $n_{\mathrm{s}}$ is the number density of the solid, and $\gamma$ is the interfacial free energy density, and the free energy has a maximum $\Delta G^{*}$ at $R=\frac{2 \gamma}{n_{\mathrm{s}}|\Delta \mu|}$ The nucleation rate per unit
volume is given by

$$
J=J_{0} \exp \left(-\Delta G^{*} / k_{\mathrm{B}} T\right)=J_{0} \exp \left[\frac{-16 \pi \gamma^{3}}{3\left(n_{\mathrm{s}}|\Delta \mu|\right)^{2} k_{\mathrm{B}} T}\right]
$$

For hard spheres the surface tension $\gamma$ should be of order $k_{\mathrm{B}} T / a^{2}$. Crystal growth in the case of reaction-limited growth should follow the Wilson-Frenkel growth law

$$
v=v_{\infty}\left[1-\exp \left(-|\Delta \mu| k_{\mathrm{B}} T\right)\right]
$$

where $v_{\infty}$ represents the growth velocity if $|\Delta \mu|$ were infinite. Comparison between theory and experiment is very challenging because of the strong dependence of the nucleation rate on $\gamma$ and $|\Delta \mu|$ as well as the need to determine the kinetic prefactor $J_{0}$. Pioneering computer simulations have been carried out to determine both the shape and height of the nucleation barrier and the kinetic prefactor $J_{0}{ }^{184-186}$ This has made possible a more careful, quantitative comparison between experiment and theory.

Experiments on colloidal crystals have employed light scattering as well as real-space techniques. The model systems must be density matched as well as refractive-index matched. Varying the colloid and solvent materials parameters to achieve this constrains one's control over other interactions. It should thus be noted that "the nearly-hard-sphere-like" colloids discussed here in fact have interparticle potentials that have a weak repulsion that is longer in range than the hard core repulsion.

In time-resolved static light scattering, the growth of the main Bragg peak is monitored during crystallization. Lightscattering experiments on hard-sphere-like colloidal suspensions $^{8,187}$ show that, below the melting volume fraction of $\phi=$ 0.545 for hard spheres, crystallization is compatible with the formation of isolated nuclei followed by growth. Above, however, crystal growth is suppressed by very high nucleation rates. At even higher volume fractions in these suspensions (with a $5 \%$ size polydispersity), the onset of the glass transition slows down all kinetics.

Microscopy studies of nucleation were carried in a colloidal suspension of spheres that were almost hard-sphere-like ${ }^{11}$ (Fig. 7A). Contrary to expectations, the nuclei observed were not spherical. The number of nuclei was determined as a function of nucleus surface area $A$, and approximating the nucleus as an ellipsoid, the solid-fluid surface tension was estimated (via a fit to the functional form $N(A)=$ $\left.\exp \left[-\gamma A / k_{\mathrm{B}} T\right]\right)$ at $\gamma=0.03 k_{\mathrm{B}} T / a^{2}$ ( $a$ being the particle radius), which is a surprisingly low value.

Crystal growth in hard spheres has been monitored via the time-dependence of the small angle light scattering peak intensity. ${ }^{188}$ Two clear regimes of growth were observed, with the early growth regime corresponding closely to the $t^{1 / 2}$ behaviour expected for a non-conserved order parameter. ${ }^{189}$ Kinetics of crystallization in charged spheres has been studied extensively as well. ${ }^{9,190}$ Crystallite growth velocities were measured and compared with the Wilson-Frenkel growth law. While the determination of the prefactor is again difficult, the functional form observed is consistent with the W-F form.

Polydispersity qualitatively alters the nucleation process. ${ }^{191}$ The structure factor displays first a broad peak which eventually (and excruciatingly slowly) evolves into an rhcp structure. This slowness allows enough time for some form of crystalline reorganization in the intermediate stages. In binary mixtures of charged spheres, ${ }^{192}$ no systematic dependence on composition is seen in the nucleation rate, indicating that random substitutional crystals nucleate in a manner similar to pure crystals: that is, charge effects swamp the effect of size disparity.

Of course, both classical nucleation theory and simulations are predicated on the assumption of homogeneous nucleation of the crystal (nucleation in the bulk without any surface to lower the nucleation barrier). Most experimenters have to work very hard to achieve homogeneous nucleation. Indeed, an additional difficulty in the study of nucleation in colloidal systems is that most colloidal crystals are prepared, then shearmelted and the nucleation process is typically recrystallization from the shear melt. It is, therefore, impossible to completely rule out the existence of tiny crystallites that alter the nucleation kinetics drastically, except in cases where one can cross a true phase transition threshold (this has been achieved for thermosensitive microgel colloids ${ }^{194}$ ).

Given these difficulties, it is perhaps not surprising that large discrepancies between simulation and experiment are found even in the simplest case of hard spheres (the $y$-axis in Fig. 7(B) and $(\mathrm{C})$ is plotted on a log scale). Theoretically, going beyond classical nucleation theory is difficult, although work in this direction is active, ${ }^{193}$ and a quantitative fit to the data of ref. 9 has been achieved (Fig. 7B). Nucleation rates are sensitively dependent on polydispersity ${ }^{185}$ and even weak electrostatic interparticle interactions. Finally, Brownian dynamics simulations show that nucleation in the presence of very weak shear ${ }^{195,196}$ increases the size of the critical nucleus and suppresses the nucleation rate. Thus nucleation under shear can provide another systematic way for a quantitative study of crystal nucleation.

A relatively clean way to study heterogenous nucleation is via the introduction of spherical (seed) impurities. Monte Carlo simulation studies of colloidal suspensions at volume fractions slightly above the freezing volume fraction $\phi=0.494$ show a nucleation rate that is expectedly much enhanced from 

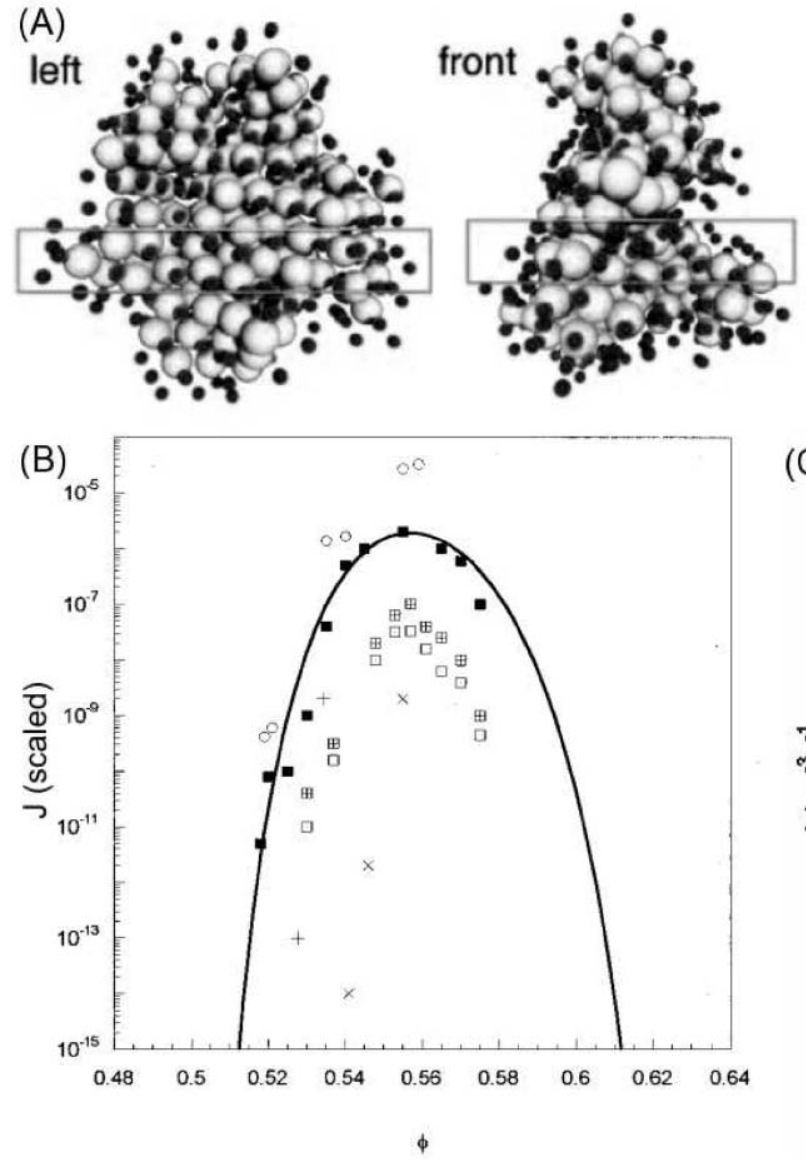

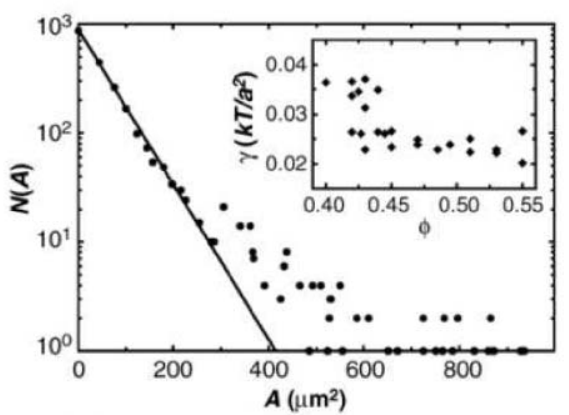

(C)

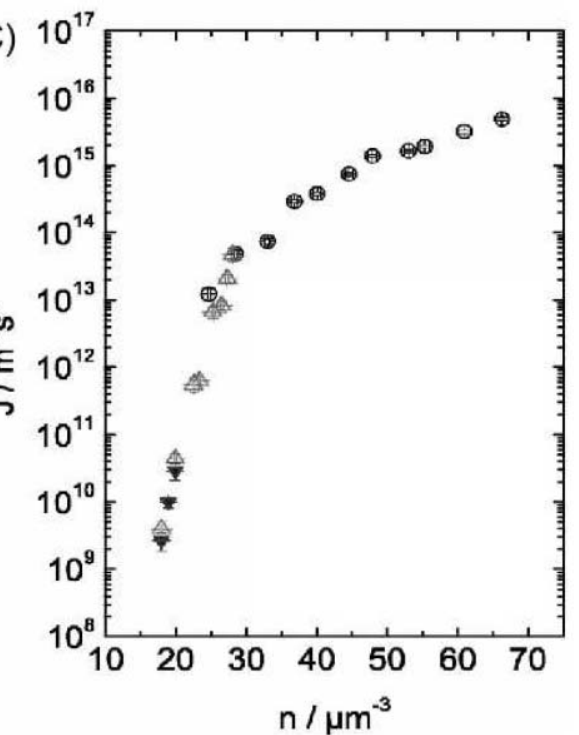

Fig. 7 (A) Confocal micrographs of a colloidal crystal nucleus composed of weakly-charged (almost hard-sphere-like) colloids. ${ }^{11}$ "Left" and "front" denote different cuts of the same crystallite, and particles with non-crystal-like bond orientational order (coloured dark) are drawn reduced in size. Both the apparent non-sphericity and the slope of a plot of number of nuclei $N(A) v s$. nucleus surface area $A$ (right) implies an anomalously low surface tension $\gamma$ (right, inset). From ref. 11. Reprinted with permission from AAAS. (B) A theoretical form for the scaled nucleation rate $J^{193}$ agrees with some, ${ }^{9}$ but not all experiments (note that the $y$-axis is a log scale!), and neither with computer simulation studies ${ }^{184-186}$ where both the shape and height of the nucleation barrier and the kinetic prefactor have been determined. Reprinted with permission from ref. 193. Copyright by the American Physical Society. http://link.aps.org/abstract/PRE/v64/e041604. (C) Time-resolved static light scattering studies in charged colloids $^{9,190}$ find nucleation rates that are consistent with classical nucleation theory, as well as growth velocities that have a functional form consistent with the classical crystal growth formula for reaction-limited growth. A quantitative comparison of theory with experiment for charged colloids awaits. Reused with permission from Patrick Wette, Hans Joachim Schope and Thomas Palberg, Journal of Chemical Physics, 123, 174902 (2005). Copyright 2005, American Institute of Physics.

the homogeneous nucleation rate, but only above a threshold value of $\approx 8$ of the seed-particle radius ratio. ${ }^{197}$ The effect of spherical impurities on (heterogeneous) nucleation and crystal growth was studied experimentally in ref. 198. Experimentally too, it was seen that homogeneous nucleation was prevalent above a threshold seed-particle radius ratio-experimentally this threshold value was between 13 and 27 .

\subsection{Crystal growth}

Growth of colloidal crystals is important technologically in their uses as photonic crystals, ${ }^{29,199}$ optically-controlled switches $^{200}$ or sensors, ${ }^{201}$ and is a topic worthy of separate review. As discussed in section 1, the growth of crystals depends in a sensitive way on the gravitational interaction, crystallizing in the microgravity of space, but not in the milligravity on Earth. Crystal growth has also been shown to be controllable via temperature gradients ${ }^{202}$ as well as a host of other techniques - shear, ${ }^{160,166}$ electric fields, ${ }^{133,134,156,157,203}$ electrochemical growth ${ }^{204}$ and vertical deposition. ${ }^{205}$

\section{Conclusions}

Colloidal phase behaviour shows a rich diversity of selfassembled mesophases. This diversity, coupled with the ability to control colloidal interparticle interactions, makes it possible to study the relationship between interactions and phase behaviour. Tunability allows the tweaking of colloidal interactions on the fly, making it possible to cycle across phase transitions. This has potentially-important applications in making advanced materials. Moreover, colloids provide a robust platform upon which to study the fundamental problem of crystal nucleation and growth, as well as other important problems in condensed matter physics, such as the glass transition. Once again, the ability to cycle controllably across 
phase transitions can provide a means to study these problems better. Tunability will offer up many new phase boundaries to cross, and control parameters to traverse the crossings.

\section{Acknowledgements}

I acknowledge numerous fruitful discussions over the years with Alfons van Blaaderen (and his research group), and Ivan Saika-Voivod and Amit Agarwal for stimulating discussions and a critical reading of the manuscript. This work was supported by the Natural Sciences and Engineering Research Council of Canada.

\section{References}

1 J. B. Perrin, Nobel Lectures on Physics 1922-1941, World Scientific, Singapore, 1998.

2 P. Pieranski, Phys. Rev. Lett., 1980, 45, 569.

3 P. N. Pusey, Liquids, freezing and glass transition, 1989, ed. J. P. Hansen, D. Levesque and J. Zinn-Justin, Elsevier Science Pub. Co., Amsterdam, New York, 1991, ch. 10.

4 K. Zahn, R. Lenke and G. Maret, Phys. Rev. Lett., 1999, 82, 2721.

5 J. A. Weiss, D. W. Oxtoby, D. G. Grier and C. A. Murray, J. Chem. Phys., 1995, 103, 1180.

6 U. Dassanayake, S. Fraden and A. van Blaaderen, J. Chem. Phys., 2000, 112, 3851.

7 A. Yethiraj and A. van Blaaderen, Nature, 2003, 421, 513.

8 J. L. Harland and W. van Megen, Phys. Rev. E, 1997, 55, 3054.

9 T. Palberg, J. Phys.: Condens. Matter, 1999, 11, R323.

10 D. Grier and C. A. Murray, J. Chem. Phys., 1994, 100, 9088.

11 U. Gasser, E. R. Weeks, A. Schofield, P. N. Pusey and D. A. Weitz, Science, 2001, 292, 258.

12 Z. Cheng, P. M. Chaikin, J. Zhu, W. B. Russel and W. V. Meyer, Phys. Rev. Lett., 2002, 88, 015501.

13 V. N. Manoharan, M. T. Elsesser and D. J. Pine, Science, 2003, 301, 483.

14 M. E. Leunissen, C. G. Christova, A.-P. Hynninen, C. P. Royall, A. I. Campbell, A. Imhof, M. Dijkstra, R. van Roij and A. van Blaaderen, Nature, 2005, 437, 235.

15 P. Bartlett and A. I. Campbell, Phys. Rev. Lett., 2005, 95, 128302.

16 W. B. Russel, D. A. Saville and W. R. Schowalter, Colloidal Dispersions, Cambridge University Press, Cambridge, England, 1989

17 A. K. Sood, Solid State Phys., 1991, 45, 73.

18 A. van Blaaderen and P. Wiltzius, Science, 1995, 270, 1177

19 E. B. Sirota, H. D. Ou-yang, S. K. Sinha, P. M. Chaikin, J. D. Axe and Y. Fujii, Phys. Rev. Lett., 1989, 62, 1524.

20 L. B. Chen, M. K. Chow, B. J. Ackerson and C. F. Zukoski, Langmuir, 1994, 10, 2817.

21 D. J. Pine, D. A. Weitz, P. M. Chaikin and E. Herbolzheimer, Phys. Rev. Lett., 1988, 60, 1134.

22 P. N. Segrè, W. van Megen, P. N. Pusey, K. Schätzel and W. Peters, J. Mod. Opt., 1995, 42, 1929.

23 T. G. Mason and D. A. Weitz, Phys. Rev. Lett., 1995, 74, 1250.

24 J. G. Kirkwood, J. Chem. Phys., 1939, 7, 919.

25 B. J. Alder and T. E. Wainwright, J. Chem. Phys., 1957, 27, 1208.

26 B. J. Alder, W. G. Hoover and D. A. Young, J. Chem. Phys., 1968, 49, 3688 .

27 P. N. Pusey and W. van Megen, Nature, 1986, 320, 340.

28 M. A. Rutgers, J. H. Dunsmuir, J. Z. Xue, W. B. Russel and P. M. Chaikin, Phys. Rev. B, 1996, 53, 5043.

29 J. D. Joannopoulos, P. R. Villeneuve and S. Fan, Nature, 1997, 386, 143.

30 A. P. Gast and C. F. Zukoski, Adv. Colloid Interface Sci., 1989, 30, 153.

31 H. Block and J. P. Kelly, J. Phys. D, 1988, 21, 1661.

32 P. N. Bartlett, M. A. Ghanem, I. S. El Hallag, P. de Groot and A. Zhukov, J. Mater. Chem., 2003, 13, 2596.

33 A. van Blaaderen, R. Ruel and P. Wiltzius, Nature, 1997, 385, 321.

34 D. van Winkle and C. A. Murray, J. Chem. Phys., 1988, 89, 3885.
35 J. P. Hoogenboom, A. K. van Langen-Suurling, J. Romijn and A. van Blaaderen, Phys. Rev. Lett., 2003, 90, 138301.

36 J. P. Hoogenboom, A. K. van Langen-Suurling, J. Romijn and A. van Blaaderen, Phys. Rev. E, 2004, 69, 051602.

37 N. V. Dziomkina and G. J. Vansco, Soft Matter, 2005, 1, 265.

38 B. J. Ackerson, S. E. Paulin, B. Johnson, W. van Megen and S. Underwood, Phys. Rev. E, 1999, 59, 6903.

39 S. I. Henderson and W. van Megen, Phys. Rev. Lett., 1998, 80 877.

40 P. N. Pusey, W. van Megen, P. Bartlett, B. J. Ackerson, J. G. Rarity and S. M. Underwood, Phys. Rev. Lett., 1989, 63, 2753.

41 P. Bartlett, P. N. Pusey and R. H. Ottewill, Langmuir, 1991, 7, 213.

42 J. Zhu, M. Li, W. Meyer, R. H. Ottewill, STS-73 Space Shuttle Crew, W. B. Russel and P. M. Chaikin, Nature, 1997, 387, 883.

43 S.-C. Mau and D. A. Huse, Phys. Rev. E, 1999, 59, 4396

44 S. Pronk and D. Frenkel, Phys. Rev. Lett., 2003, 255, 501.

45 J. K. G. Dhont, An Introduction to Dynamics of Colloids, Elsevier, Amsterdam, 1996.

46 G. K. Batchelor, J. Fluid Mech., 1972, 52, 245

47 H. Hayakawa and K. Ichiki, Phys. Rev. E, 1995, 51, R3815.

48 J. T. Padding and A. A. Louis, Phys. Rev. Lett., 2004, 93, 220601.

49 R. E. Caflisch and J. H. C. Luke, Phys. Fluids, 1985, 28, 759.

50 S. Ramaswamy, Adv. Phys., 2001, 50, 297.

51 S.-Y. Tee, P. J. Mucha, L. Cipelletti, S. Manley, M. P. Brenner, P. N. Segre and D. A. Weitz, Phys. Rev. Lett., 2002, 89, 054501.

52 X. Lei, B. J. Ackerson and P. Tong, Phys. Rev. Lett., 2001, 86, 3300.

53 J. H. Page, M. L. Cowan and D. A. Weitz, Physica B, 2000, 279, 130.

54 P. N. Segrè, E. Herbolzheimer and P. M. Chaikin, Phys. Rev Lett., 1997, 79, 2574.

55 D. L. Koch and E. S. G. Shaqfeh, J. Fluid Mech., 1991, 224, 275.

56 A. Levine, S. Ramaswamy, E. Frey and R Bruinsma, Phys. Rev. Lett., 1998, 81, 5944.

57 J. H. C. Luke, Phys. Fluids, 2000, 12, 1619

58 D. O. Riese, G. H. Wegdam, W. L. Vos, R. Sprik, D. Fenistein, J. H. H. Bongaerts and G. Grubel, Phys. Rev. Lett., 2000, 85, 5460.

59 M. P. Brenner, Phys. Fluids, 1999, 11, 754.

60 D. Rudhardt, C. Bechinger and P. Leiderer, Phys. Rev. Lett. 1998, 81, 1330 .

61 A. P. Gast, C. K. Hall and W. B. Russel, J. Colloid Interface Sci., 1983, 96, 251.

62 S. Asakura and F. Oosawa, J. Polym. Sci., 1958, 33, 183

63 H. N. W. Lekkerkerker, W. C. K. Poon, P. N. Pusey, A. Stroobants and P. B. Warren, Europhys. Lett., 1992, 20, 559.

64 P. N. Pusey, A. D. Pirie and W. C. K. Poon, Physica A, 1993, 201, 322.

65 F. L. Calderon, J. Bibette and J. Bias, Europhys. Lett., 1993, 23 653.

66 S. M. Ilett, A. Orrock, W. C. K. Poon and P. N. Pusey, Phys. Rev E, 1995, 51, 1344

67 S. Ramakrishnan, M. Fuchs, K. S. Schweizer and C. F. Zukoski, J. Chem. Phys., 2002, 116, 2201.

68 P. N. Segrè, V. Prasad, A. B. Schofield and D. A. Weitz, Phys. Rev. Lett., 2001, 86, 6042.

69 W. C. K. Poon and M. D. Haw, Adv. Colloid Interface Sci., 1997, 73,71 .

70 D. G. A. L. Aarts, M. Schmidt and H. N. W. Lekkerkerker, Science, 2004, 304, 847

71 K. N. Pham, A. M. Peurtas, J. Bergenholtz, S. U. Egelhaaf, A. Moussaid, P. N. Pusey, A. B. Schofield, M. E. Cates, M. Fuchs and W. C. K. Poon, Science, 2002, 296, 104

72 K. Dawson, G. Foffi, M. Fuchs, W. Götze, F. Sciortino, M. Sperl, P. Tartaglia, Th. Voigtmann and E. Zaccarelli, Phys. Rev. E 2000, 63, 011401

73 A. M. Puertas, M. Fuchs and M. E. Cates, Phys. Rev. Lett., 2002, 88, 098301.

74 W. C. K. Poon, J. Phys.: Condens. Matter, 2002, 14, R859.

75 L. J. Kaufman and D. A. Weitz, J. Chem. Phys., 2006, 125, 074716 
76 S. Manley, H. M. Wyss, K. Miyazaki, J. C. Conrad, V. Trappe, L. J. Kaufman, D. R. Reichman and D. A. Weitz, Phys. Rev. Lett., 2005, 95, 238302.

77 T. Biben, P. Bladon and D. Frenkel, J. Phys.: Condens. Matter, 1996, 8, 10799.

78 J. C. Crocker, J. A. Matteo, A. D. Dinsmore and A. G. Yodh, Phys. Rev. Lett., 1999, 82, 4352.

79 M. Dijkstra, R. van Roij and R. Evans, Phys. Rev. E, 1999, 59, 5744.

80 A. D. Dinsmore, A. G. Yodh and D. J. Pine, Phys. Rev. E, 1995, 52, 4045 .

81 P. D. Kaplan, A. G. Yodh and D. J. Pine, Phys. Rev. Lett., 1992, 68, 393 .

82 S. Sanyal, N. Easwar, S. Ramaswamy and A. K. Sood, Europhys. Lett., 1992, 18, 107.

83 H. N. W. Lekkerkerker and A. Stroobants, Physica A, 1993, 195, 387.

84 A. Imhof and J. K. G. Dhont, Phys. Rev. Lett., 1995, 75, 1662.

85 P. Bartlett, R. H. Ottewill and P. N. Pusey, Phys. Rev. Lett., 1992, 68, 3801.

86 K. P. Velikov, C. G. Christova, R. P. A. Dullens and A. van Blaaderen, Science, 2002, 296, 106.

87 R. Roth and M. Kinoshita, J. Chem. Phys., 2006, 125, 084910.

88 K. Kremer, M. O. Robbins and G. S. Grest, Phys. Rev. Lett., 1986, 57, 2694

89 M. O. Robbins, K. Kremer and G. S. Grest, J. Chem. Phys., 1988, 88, 3286.

90 E. J. Meijer and D. Frenkel, J. Chem. Phys., 1991, 94, 2269

91 E. J. Meijer and F. El Azhar, J. Chem. Phys., 1997, 106, 4678.

92 J.-P. Hansen and L. Verlet, Phys. Rev., 1969, 184, 151.

93 F. A. Lindemann, Phys. Z., 1910, 11, 609.

94 Y. Monovoukas and A. P. Gast, J. Colloid Interface Sci., 1989, 128, 533.

95 C. P. Royall, M. E. Leunissen and A. van Blaaderen, J. Phys.: Condens. Matter, 2003, 15, S3581.

96 G. M. Kepler and S. Fraden, Phys. Rev. Lett., 1994, 73, 356.

97 A. E. Larsen and D. G. Grier, Nature, 1997, 385, 230.

98 B. V. R. Tata, E. Yamahara, P. V. Rajamani and N. Ise, Phys. Rev. Lett., 1997, 78, 2660.

99 R. Rajagopalan and K. Srinivasa Rao, Phys. Rev. E, 1997, 55, 4423.

100 K. S. Rao and R. Rajagopalan, Phys. Rev. E, 1998, 57, 3227.

101 E. R. Dufresne, T. M. Squires, M. P. Brenner and D. G. Grier, Phys. Rev. Lett., 2000, 85, 3317.

102 S. H. Behrens and D. G. Grier, Phys. Rev. E, 2001, 64, 050401R.

103 M. Medebach, R. C. Jordan, H. Reiber, H.-J. Schope, R. Biehl, M. Evers, D. Hessinger, J. Olah, T. Palberg, E. Schonberger and P. Wette, J. Chem. Phys., 2005, 123, 104903.

104 D. F. Evans and H. Wennerstrom, The Colloidal Domain: Where Physics, Chemistry, Biology and Technology Meet, 2nd edn, WileyVCH, 1999, ch. 8

105 M. Brunner, J. Dobnikar, H. H. von Grünberg and C. Bechinger, Phys. Rev. Lett., 2004, 92, 078301.

106 R. Klein, H. H. von Grünberg, C. Bechinger, M. Brunner and V. Lobashkin, J. Phys.: Condens. Matter, 2002, 14, 7631

107 A.-P. Hynninen, M. Dijkstra and R. van Roij, Phys. Rev. E, 2004, 69, 061407.

108 H. J. Schöpe, T. Decker and T. Palberg, J. Chem. Phys., 1998, 109, 10068

109 A. M. Kalsin, M. Fialkowski, M. Paszewski, S. K. Smoukov, K. J. M. Bishop and B. A. Grzybowski, Science, 2006, 312, 420.

110 K. M. Ho, C. T. Chan and C. M. Soukoulis, Phys. Rev. Lett., 1990, 65, 3152

111 M. Maldovan and E. L. Thomas, Nat. Mater., 2004, 3, 593.

112 H. Lowen, J. Phys.: Condens. Matter, 2001, 13, R415.

113 A. van Blaaderen, MRS Bull., 2004, 29, 85.

114 M. Parthasarathy and D. J. Klingenberg, Mater. Sci. Eng., R, 1996, 17, 57

115 J. M. Ginder, MRS Bull., 1998, 23, 26.

116 Y. Hu, J. L. Glass, A. E. Griffith and S. Fraden, J. Chem. Phys, 1994, 100, 4674

117 T. C. Halsey and W. Toor, Phys. Rev. Lett., 1990, 65, 2820.

118 J. E. Martin, J. Odinek, T. C. Halsey and R. Kamien, Phys. Rev. $E, \mathbf{1 9 9 8 , 5 7 , 7 5 6 .}$

119 R. Tao and J. M. Sun, Phys. Rev. Lett., 1991, 67, 398.
120 R. Tao, Phys. Rev. E, 1993, 47, 423.

121 A. Yethiraj and A. van Blaaderen, Int. J. Mod. Phys. B, 2002, 16, 2328

122 A. Yethiraj and A. van Blaaderen, unpublished.

123 A. Kumar, B. Khusid, Z. Qiu and A. Acrivos, Phys. Rev. Lett., $2005,95,258301$

124 W. J. Wen, X. X. Huang, S. H. Yang, K. Q. Lu and P. Sheng, Nat. Mater., 2003, 2, 727.

125 W. Wen, X. Huang and P. Sheng, Appl. Phys. Lett., 2004, 85, 299.

126 A. Yethiraj, A. Wouterse, B. Groh and A. van Blaaderen, Phys. Rev. Lett., 2004, 92, 058301.

127 P. J. Rankin, J. M. Ginder and D. J. Klingenberg, Curr. Opin. Colloid Interface Sci., 1998, 3, 373

128 D. R. Nelson and B. I. Halperin, Phys. Rev. B, 1979, 19, 2457.

129 J. M. Kosterlitz and D. J. Thouless, J. Phys. C, 1972, 5, L124.

130 K. Zahn and G. Maret, Phys. Rev. Lett., 2000, 85, 3656.

131 S. H. L. Klapp, J. Phys.: Condens. Matter, 2005, 17, R525.

132 A. D. Hollingsworth and D. A. Saville, J. Colloid Interface Sci., 2004, 272, 235.

133 M. Trau, S. Sankaran, D. A. Saville and I. A. Aksay, Langmuir, 1995, 11, 4665.

134 M. Trau, I. A. Aksay and D. A. Saville, Science, 1996, 272, 706.

135 D. L. Blair, T. Neicu and A. Kudrolli, Phys. Rev. E, 2003, 67, 031303 .

136 A. E. Cohen and W. E. Moerner, Proc. Natl. Acad. Sci. U. S. A., 2006, 103, 4362

137 R. Pethig and G. H. Markx, Trends Biotechnol., 1997, 15, 426.

138 A. Ashkin, J. M. Dziedzic, J. E. Bjorkholm and S. Chu, Opt. Lett., 1986, 11, 288.

139 A. Ashkin and J. M. Dziedzic, Proc. Natl. Acad. Sci. U. S. A., 1989, 86, 7914.

140 A. Ashkin, K. Schutze, J. M. Dziedzic, U. Euteneuer and M. Schliwa, Nature, 1990, 348, 346

141 M. Reicherter, T. Haist, E. U. Wagemann and H. J. Tiziani, Opt. Lett., 1999, 24, 608.

142 J. E. Curtis, B. A. Koss and D. G. Grier, Opt. Commun., 2002, 207, 169.

143 W. J. Hossack, E. Theofanidou, J. Crain, K. Heggarty and M. Birch, Opt. Express, 2003, 11, 2053.

144 D. G. Grier and Y. Roichman, Appl. Opt., 2006, 45, 880.

145 B. Agate, C. T. A. Brown, W. Sibbett and K. Dholakia, Opt. Express, 2004, 12, 3011.

146 D. L. J. Vossen, A. van der Horst, M. Dogterom and A. van Blaaderen, Rev. Sci. Instrum., 2004, 75, 2960.

147 E. M. Furst, Soft Mater, 2003, 1, 167.

148 C. Bechinger, Curr. Opin. Colloid Interface Sci., 2002, 7, 204.

149 Q.-H. Wei, C. Bechinger and P. Leiderer, Science, 2000, 287, 625.

150 C. Bechinger, M. Brunner and P. Leiderer, Phys. Rev. Lett., 2001, 86, 930.

151 S.-H. Lee and D. G. Grier, Phys. Rev. Lett., 2006, 96, 190601.

152 A. Pertsinidis and X. S. Ling, Nature, 2001, 413, 147.

153 Y. Roichman and D. G. Grier, Opt. Express, 2005, 13, 5434.

154 R. Krupke, F. Hennrich, H. von Lohneysen and M. M. Kappes, Science, 2003, 301, 344.

155 M. T. Sullivan, K. Zhao, A. D. Hollingsworth, R. H. Austin, W. B. Russel and P. M. Chaikin, Phys. Rev. Lett., 2006, 96, 015703.

156 K. H. Bhatt and O. D. Velev, Langmuir, 2004, 20, 467.

157 K. H. Bhatt, S. Grego and O. D. Velev, Langmuir, 2005, 21, 6603.

158 S. Butler and P. Harrowell, Phys. Rev. Lett., 1995, 52, 6424.

159 B. J. Ackerson and N. A. Clark, Phys. Rev. Lett., 1981, 46, 123.

160 P. Holmqvist, M. P. Lettinga, J. Buitenhuis and J. K. G. Dhont, Langmuir, 2005, 21, 10976.

161 R. Biehl and T. Palberg, Europhys. Lett., 2004, 66, 291.

162 I. Cohen, T. G. Mason and D. A. Weitz, Phys. Rev. Lett., 2004, 93, 046001.

163 J. Guery, E. Bertrand, C. Rouzeau, P. Levitz, D. A. Weitz and J. Bibette, Phys. Rev. Lett., 2006, 96, 198301.

164 S. Butler and P. Harrowell, Nature, 2002, 415, 1008

165 S. Butler and P. Harrowell, J. Chem. Phys., 2003, 118, 4115.

166 P. Jiang and M. J. McFarland, J. Am. Chem. Soc, 2004, 126, 13778.

167 J. C. Loudet, P. Poulin and P. Barois, Europhys. Lett., 2001, 54, 175. 
168 P. Poulin, V. A. Raghunathan, P. Richetti and D. Roux, J. Phys. II, 1994, 4, 1557.

169 V. A. Raghunathan, P. Richetti, D. Roux, F. Nallet and A. K. Sood, Langmuir, 2000, 16, 4720.

170 J. C. Loudet, Liq. Cryst. Today, 2004, 14, 1.

171 J. C. Loudet, P. Barois, P. Auroy, P. Keller, H. Richard and P. Poulin, Langmuir, 2004, 20, 11336.

172 D. Andrienko, M. P. Allen, G. Skačej and S. Zumer, Phys. Rev. E, 2002, 65, 041702 .

173 T. G. Sokolovska, R. O. Sokolovskii and G. N. Patey, Phys. Rev. E, 2006, 73, 020701R.

174 P. Poulin, H. Stark, T. C. Lubensky and D. A. Weitz, Science, 1997, 275, 1770.

175 S. P. Meeker, W. C. K. Poon, J. Crain and E. M. Terentjev, Phys. Rev. E, 2000, 61, R6083.

176 J. Arrault, C. Grand, W. C. K. Poon and M. E. Cates, Europhys. Lett., 1997, 38, 625.

177 P. Poulin, V. Cabuil and D. A. Weitz, Phys. Rev. Lett., 1997, 79, 4862.

178 H. Lowen, et al., J. Phys.: Condens. Matter, 2005, 17, S3379.

179 M. P. Lettinga, H. Wang and J. K. G. Dhont, Phys. Rev. E, 2004, 70, 061405.

180 D. Derks, et al., Phys. Rev. Lett., 2006, 97, 038301.

181 E. V. Shevchenko, D. V. Talapin, N. A. Kotov, S. O'Brien and C. B. Murray, Nature, 2006, 439, 55.

182 N. D. Denkov, O. D. Velev, P. A. Kralchevsky, I. B. Ivanov, J. H. Yoshimura and K. Nagayama, Langmuir, 1992, 8, 3183.

183 J. Dzubiella, G. P. Hoffmann and H. Lowen, Phys. Rev. E, 2002, 65, 021402 .

184 S. Auer and D. Frenkel, Nature, 2001, 409, 1020.

185 S. Auer and D. Frenkel, Nature, 2001, 413, 711.

186 S. Auer and D. Frenkel, J. Phys.: Condens. Matter, 2002, 14, 7667.
187 J. L. Harland, S. I. Henderson, S. M. Underwood and W. van Megen, Phys. Rev. Lett., 1995, 75, 3572.

188 K. Schatzel and B. J. Ackerson, Phys. Rev. E, 1993, 48, 3766.

189 S. M. Allen and J. W. Cahn, Acta Metall., 1979, 27, 1085.

190 P. Wette, H. J. Schope and T. Palberg, J. Chem. Phys., 2005, 123, 174902.

191 H. J. Schope, G. Bryant and W. van Megen, Phys. Rev. Lett., 2006, 96, 175701.

192 P. Wette, H. J. Schope and T. Palberg, J. Chem. Phys., 2005, 122, 144901.

193 N. M. Dixit and C. F. Zukoski, Phys. Rev. E, 2001, 64, 041604.

194 S. Tang, Z. Hu, Z. Cheng and J. Wu, Langmuir, 2004, 20, 8858.

195 R. Blaak, S. Auer, D. Frenkel and H. Lowen, Phys. Rev. Lett., 2004, 93, 068303.

196 R. Blaak, S. Auer, D. Frenkel and H. Lowen, J. Phys.: Condens. Matter, 2004, 16, S3873.

197 A. Cacciuto, S. Auer and D. Frenkel, Nature, 2004, 428, 404.

198 V. W. A. de Villeneuve, D. Verboekend, R. P. A. Dullens, D. G. A. L. Aarts, W. K. Kegel and H. N. W. Lekkerkerker, J. Phys.: Condens. Matter, 2005, 17, S3371.

199 K. Busch and S. John, Phys. Rev. E, 1998, 58, 3896B.

200 G. Pan, R. Kesavamoorthy and S. A. Asher, Phys. Rev. Lett., 1997, 78, 3860.

201 J. H. Holtz and S. A. Asher, Nature, 1997, 389, 829.

202 Z. D. Cheng, W. B. Russell and P. M. Chaikin, Nature, 1999, 401, 893.

203 A. Yethiraj, J. H. J. Thijssen, A. Wouterse and A. van Blaaderen, Adv. Mater., 2004, 16, 596.

204 P. V. Braun and P. Wiltzius, Nature, 1999, 402, 603.

205 P. Jiang, J. F. Bertone, K. S. Hwang and V. L. Colvin, Chem. Mater., 1999, 11, 2132. 